\title{
ALENDRONATE REDUCED PERI-TUNNEL BONE LOSS AND ENHANCED TENDON GRAFT TO BONE TUNNEL HEALING IN ANTERIOR CRUCIATE LIGAMENT RECONSTRUCTION
}

\author{
P.P.Y. Lui ${ }^{1,2,3, *}$, Y.W. Lee ${ }^{1,2}$, T.Y. Mok ${ }^{1,2}$, Y.C. Cheuk ${ }^{1,2}$ and K.M. Chan ${ }^{1,2,3}$ \\ ${ }^{1}$ Department of Orthopaedics and Traumatology, Faculty of Medicine, The Chinese University of Hong Kong, \\ Hong Kong SAR, China. \\ ${ }^{2}$ The Hong Kong Jockey Club Sports Medicine and Health Sciences Centre, Faculty of Medicine, \\ The Chinese University of Hong Kong, Hong Kong SAR, China. \\ ${ }^{3}$ Program of Stem Cell and Regeneration, School of Biomedical Science, The Chinese University of Hong Kong, \\ Hong Kong SAR, China.
}

\begin{abstract}
Peri-tunnel bone loss after anterior cruciate ligament (ACL) reconstruction is commonly observed, both clinically and experimentally. We aimed to study the effect and mechanisms of different doses of alendronate in the reduction of peritunnel bone loss and promotion of graft-bone tunnel healing in ACL reconstruction. Eighty-four ACL-reconstructed rats were divided into 4 groups. Alendronate at different dosages, or saline, were injected subcutaneously weekly, for 2 or 6 weeks post-reconstruction, for vivaCT (computed tomography) imaging, biomechanical tests, histology and immunohistochemistry. Alendronate significantly increased bone mass and density of tissue inside bone tunnels except at the epiphyseal region of tibial tunnel. The femoral tunnel diameter decreased significantly in the mid-dose and highdose alendronate groups compared to that in the saline group at week 6 . Alendronate significantly increased the peri-tunnel bone mass and density along all tunnel regions at week 6. Better graft-bone tunnel integration and intra-tunnel graft integrity were observed in the alendronate groups. The ultimate load was significantly higher in the mid-dose and high-dose alendronate groups at week 2, but not at week 6 . There was a reduction in matrix metalloprotein (MMP)1, MMP13 and CD68-positive cells at the peri-tunnel region and graft-bone interface in the alendronate-treated group compared to the saline group. Alendronate reduced peritunnel bone resorption, increased mineralised tissue inside bone tunnel as well as histologically and biomechanically promoted graft-bone tunnel healing, probably by reducing the expression of MMP1, MMP13 and CD68-positive cells. Alendronate might be used for reducing peri-tunnel bone loss and promoting graft-bone tunnel healing at early stage post-ACL reconstruction.
\end{abstract}

Keywords: Anterior cruciate ligament reconstruction; tendon graft to bone tunnel healing; bisphosphonate; alendronate; peri-tunnel bone loss.

*Address for correspondence:

Pauline Po Yee Lui

Rm. 74025, 5/F, Clinical Sciences Building

Prince of Wales Hospital

Shatin, Hong Kong Special Administrative Region, China. Telephone Number: (852)2632-3072

FAX Number: (852)2646-3020

E-mail: paulinelui00@gmail.com

\section{Introduction}

Anterior cruciate ligament (ACL) reconstruction is frequently required to restore the stability of the joint and thereby minimise injury to both the chondral surfaces and surrounding tissues. The success rate of ACL reconstructive surgery has been reported to vary from 73 to $95 \%$ and the return to pre-injury activity level has been reported to vary from 37 to $75 \%$ (Yunes et al., 2001; Fithian et al., 2005). Despite the relatively high rate of positive outcome reported, graft failure continues to occur. Such graft failure is mainly attributed to surgical errors, traumatic injuries, failure of graft osteo-integration and graft remodelling (George et al., 2006). Further, healing of harvested ACL grafts is very slow. The attachment site of grafted tendon-to-bone is the weak link in early tendon allograft/autograft to bone tunnel healing. Various methods have been reported to improve early fixation and facilitate subsequent healing of tendon graft inside bone tunnel (Fu et al., 1999; Rodeo et al., 1999; Ohtera et al., 2000; Anderson et al., 2001; Weiler et al., 2002; Lim et al., 2004; Wang et al., 2005; Ma et al., 2007).

Recent clinical studies showed that there was significant decrease in local bone mass density (BMD) around the knee region after ACL reconstruction and the decrease could persist for many years without returning to the baseline level (Kartus et al., 1998; Leppala et al., 1999; Ejerhed et al., 2004; Rittweger et al., 2005; Zerahn et al., 2006). However, these were not randomised or controlled clinical studies. Bone loss after tendon insertion site injury and repair has also been reported in animal studies (Rodeo et al., 1999; Silva et al., 2002; Ditsios et al., 2003; Galatz et al., 2005; Wen et al., 2009). There was significant reversible decrease in BMD around the knee joint as measured by dual-energy X-ray absorptiometry (DXA) in patients undergoing ACL reconstruction in a randomised controlled trial comparing bone loss with different surgical techniques (Lui et al., 2012). In a study using a rabbit ACL reconstruction model, the averaged femoral and tibial tunnel diameters did not change up to week 12 as compared to that at baseline with the progression of healing, implying that bone resorption was fast (Lui et al., 2010b).

Despite the significant decrease in BMD (Kannus et al., 1992; Kartus et al., 1998; Zerahn et al., 2006), the clinical relevance of post-operative bone loss remains unclear and evidences were inconsistent. Several authors reported that 
BMD correlated significantly with the functional scores of the injured knee after reconstruction (Kannus et al. 1992; Kartus et al. 1998; Zerahn et al. 2006). Theoretically, bone loss could have a negative impact on graft-bone tunnel healing. This is because the tunnel surface might become less stable for tendon-bone integration. The decrease in peri-tunnel BMD might also undermine the support of the graft-bone tunnel complex, complicate revision surgery and lead to degenerative joint diseases due to the production of inflammatory cytokines associated with bone resorption or even fracture due to the reduction in bone strength. Significant bone loss and decrease in mechanical properties after flexor tendon insertion site injury and repair were reported, supporting the relationship between bone loss and strength (Silva et al., 2002; Galatz et al., 2005). Prevention of excessive local bone loss due to disuse and trauma might improve tendon-to-bone tunnel healing. There was positive correlation between BMD at the distal femur and single-leg hop distance at 1 year, suggesting that BMD at the distal femur might positively affect the stability of the knee joint after reconstruction (Lui et al., 2012).

Bisphosphonates are agents that reduce bone remodelling through modulating both osteoblastic and osteoclastic activities (Giuliani et al., 1998a; Giuliani et al., 1998b; von Knoch et al., 2005). Alendronate has been reported to suppress bone loss and improve tendonto-bone repair strength in a flexor tendon canine model (Thomopoulos et al., 2007). In another study, clodronate was reported to induce macrophage apoptosis and promote tendon graft to bone tunnel healing (Hays et al., 2008). The first study was not an ACL reconstruction model and the second study used the non-nitrogen-containing bisphosphonate, which has different mechanisms of action compared to nitrogen-containing bisphosphonate. The effect of clodronate on the peri-tunnel bone was not studied (Hays et al., 2008).

We hypothesised that alendronate, a nitrogencontaining bisphosphonate, might reduce peri-tunnel bone loss and promote tendon graft to bone tunnel healing in ACL reconstruction by down-regulating the expression of matrix metalloproteinase 1 (MMP1) and MMP13 as well as inhibiting the formation of osteoclasts. This study thus aimed to study the effect of different doses of alendronate in the reduction of peri-tunnel bone loss and promotion of tendon graft to bone tunnel healing in a rat ACL reconstruction model. The expression of MMP1, MMP13, and CD68 (a monocyte-macrophage lineage marker that labels all potential osteoclast precursors and osteoclasts) at the peri-tunnel region and tendon-bone tunnel interface were also examined at the most effective alendronate dose.

\section{Materials and Methods}

\section{Animal operation}

This study was approved by the animal research ethics committee of the authors' institution. Eighty-four male Sprague Dawley rats (12 weeks, mean weight: $440 \pm 21 \mathrm{~g}$ ) were used. ACL reconstructions were performed as described previously (Lui et al., 2010b; Lui et al., 2011). Animals were sedated by intramuscular injection of $10 \%$ ketamine $/ 2 \%$ xylazine (Kethalar, $0.3 \mathrm{~mL}: 0.2 \mathrm{~mL}$ ) and sedation was maintained with $10 \%$ ketamine injected intravenously (Sigma-Aldrich, St Louis, MO, USA). The ipsilateral flexor digitorum longus tendon $(20 \mathrm{~mm}$ in length and $1 \mathrm{~mm}$ in diameter) was harvested through a longitudinal medial incision. The ACL was excised. Tibial and femoral tunnels of $1.1 \mathrm{~mm}$ in diameter and $7 \mathrm{~mm}$ in length were created from the footprint of the original ACL to the medial side of the tibia and lateral-anterior femoral condyle, respectively, with an angle of $55^{\circ}$ to the articular surface. The graft was inserted and routed through the bone tunnels, fixed on the femoral and tibial tunnel exits with a suture tied over the neighbouring periosteum at a tension of $0.7 \mathrm{~N}$ at $30^{\circ}$ of knee flexion. Soft tissue was closed in layers. Only one randomly-selected limb was operated on. $0.1 \mathrm{~mL}$ of Temgesic was injected intra-muscularly when the rat regained consciousness. The animals were allowed to have free cage movement immediately after operation. Except redness and swelling at the reconstructed site, no other postoperative complications were observed after the surgery. The rats grew as indicated by the increase in body weight with time after surgery (results not shown). There was no significant difference between the body weight of rats in the saline and alendronate groups (results not shown).

\section{Alendronate injection}

Alendronate sodium trihydrate (Sigma-Aldrich) was dissolved in $1 \mathrm{~mL}$ sterile saline and injected subcutaneously either at $6 \mu \mathrm{g} / \mathrm{kg}$ (low-dose group), $60 \mu \mathrm{g} / \mathrm{kg}$ (middle-dose group) or $300 \mu \mathrm{g} / \mathrm{kg}$ (high-dose group) once/week starting from the day after surgery for 2 or 6 weeks. Control rats received $1 \mathrm{~mL}$ saline instead. The subcutaneous dose of $60 \mu \mathrm{g} /$ $\mathrm{kg} /$ week was chosen based on the same dose used in the previous studies for the treatment of osteoporosis (Ma et al., 2003) and fracture healing (Cao et al., 2007). The other doses were chosen as $1 / 10 \mathrm{x}$ and $5 \mathrm{x}$ of this dose.

\section{Sample harvest}

At week 2 or week 6 , the animals were euthanised by overdosing (about $1 \mathrm{~mL}$ ) of $20 \%$ sodium phenobarbital injected intravenously and the reconstructed ACL complex was harvested. Nine samples per group per time point were harvested for vivaCT imaging followed immediately by biomechanical test within one month. The tendon graft to bone tunnel complexes after biomechanical test were embedded for histology and immunohistochemistry $(n=3$ per group per time point). In order to show that the tendon graft to bone tunnel complexes after biomechanical test were also suitable for histology, another 3 animals from each group at week 6 were killed and the tendon graft to bone tunnel complexes were immediately harvested for histology and compared with the histological results of samples with prior biomechanical test.

\section{VivaCT imaging and data analysis}

A cone-beam vivaCT (computed tomography) system (VivaCT40, Scanco Medical AG, Bassersdorf, Switzerland) was used to assess the bone mass and density of mineralised tissue inside the bone tunnels and the peri-tunnel bone at the knee region as described previously (Lui et al., 2010b). 

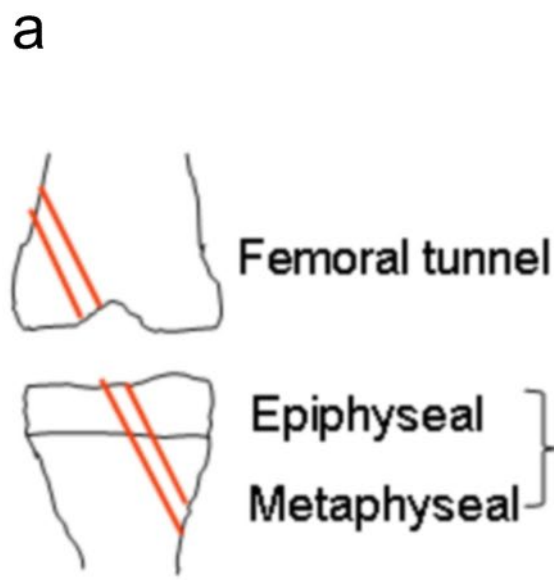

Epiphyseal

Metaphyseal

\section{Tibial tunnel}
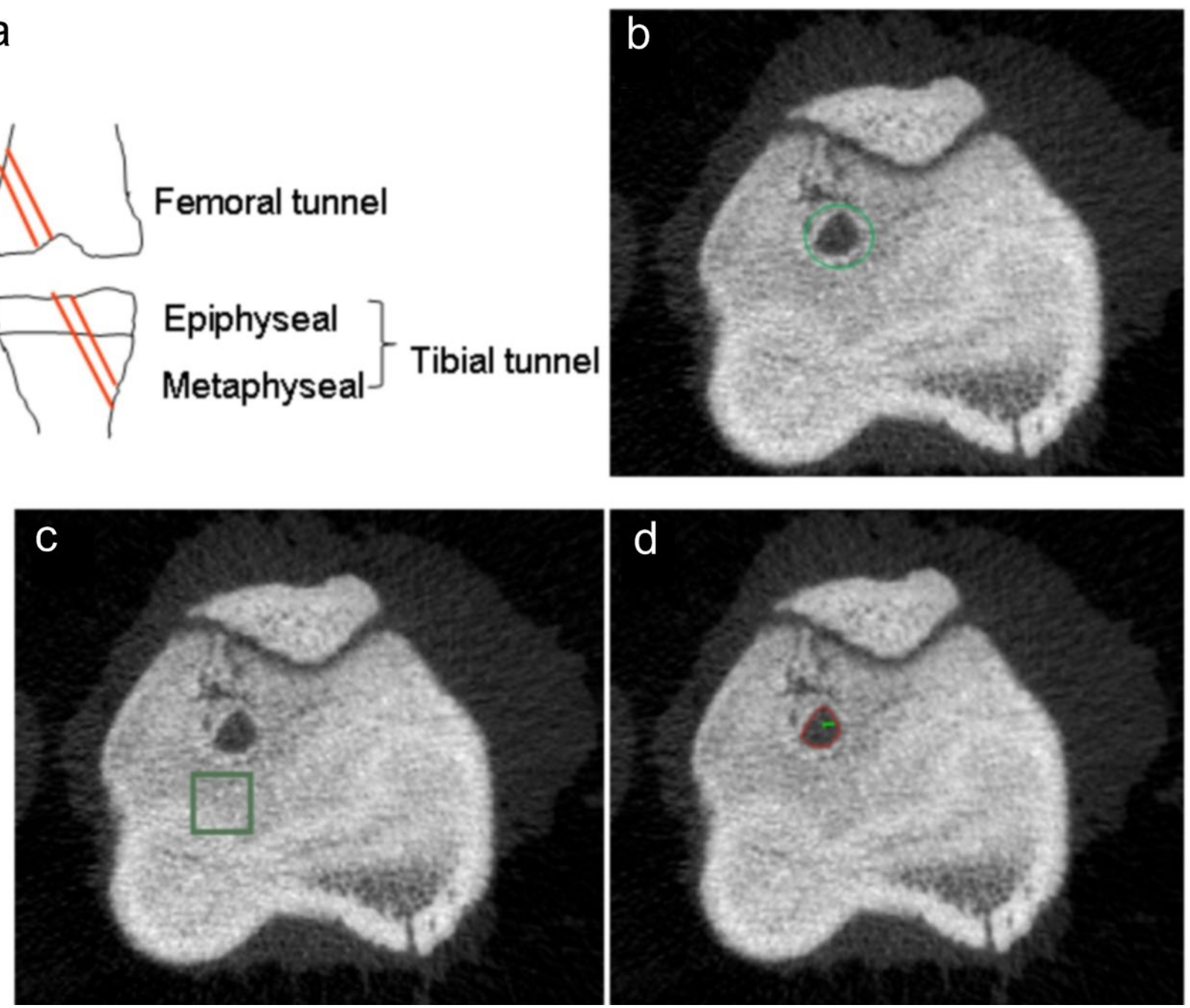

Fig. 1. vivaCT images illustrating (a) divisions of bone tunnel for the measurement of (b) mineralised tissue inside the bone tunnel (circle), (c) peri-tunnel bone (rectangle) and (d) tunnel diameter after ACL reconstruction.

The vivaCT system was regularly calibrated once per week using a phantom. All the vivaCT measurements were done by the same co-investigator with an intra-class correlation (ICC) ranging from 0.940 to 0.999 for the measurement of bone mass and density at the peri-tunnel bone and tunnel bone. After thawing the sample and removing the surrounding muscles, the region covering the entry and exit of the bone tunnel was scanned with a vertical displacement of $30 \mu \mathrm{m}$ for about 220 and 350 consecutive sections, respectively, for the femoral and tibial tunnels at the resolution of $35 \mu \mathrm{m}$. The whole process took $20 \mathrm{~min}$ and the sample was kept moist during scanning. The sample was then subjected to biomechanical tests immediately after scanning. The sections were 3-dimensionally (3D) reconstructed and rotated to align the bone tunnel vertically using the built-in software. To measure the mass and density of mineralised tissue inside the bone tunnel, a circular $1.2 \mathrm{~mm}$ region of interest (ROI) inside the bone tunnel, representing the initial tunnel diameter and confirmed by low bone volume/total volume fraction (BV/ TV) at day 0 , was then chosen and $3 \mathrm{D}$ reconstructed after thresholding to remove the signals from soft tissue (e.g., tendon graft) (Fig. 1b). To measure the peri-tunnel bone mass and density, a $0.1 \mathrm{~cm}$ x $0.1 \mathrm{~cm}$ square was chosen next to the bone tunnel in the trabecular bone region and $3 \mathrm{D}$ reconstructed after thresholding (Fig. 1c). BMD as defined by vivaCT (unit: $\mathrm{mg} \mathrm{HA} / \mathrm{ccm}$ ) and BV/TV was calculated for ROI covering the whole femoral tunnel, the epiphyseal and the metaphyseal segments of tibial tunnel (Fig. 1a). Due to its shorter length and located only in the epiphyseal region, the femoral tunnel was not further divided into 2 segments in vivaCT analysis. About 50 sections were analysed in each segment and were very consistent among different animals. vivaCT analysis was performed for 9 samples of each group at each time point. Representative 3D images matching the conclusion of image analysis were selected for presentation.

The actual tunnel diameter was also measured at the femoral tunnel as well as at the epiphyseal and metaphyseal segments of tibial tunnel using image analysis software (Image Pro Plus 6.02, Media Cybernetics, Rockville, MD, USA). To measure the bone tunnel diameter, we first exported the image with scale bar from the vivaCT system to a desktop computer. The image was then opened using the Image Pro Plus software. The function for measuring the minimum tunnel diameter was selected. The actual tunnel wall was then traced as the region of interest (Fig. 1d). The minimum tunnel diameter was then measured 
in pixels using the "Count Size" function. The length of the scale bar on the image was then measured using the "Measure length and distances" function and the actual minimum tunnel diameter in millimetres $(\mathrm{mm})$ was then calculated. The minimum tunnel diameters of four equallyspaced sections in each bone tunnel segment were averaged. The actual tunnel diameter was measured for 9 samples of each group at each time point. The same co-investigator performed all the tunnel diameter measurements with an ICC of 0.998 (95 \% CI: 0.994-1.000).

\section{Biomechanical testing}

After vivaCT scanning, the two bone ends of the femurgraft-tibia construct were placed in polyethylene tubes and potted in methyl-methacrylate. The samples were then stripped of all soft tissues, including the capsule and other knee ligaments. Cross pins were passed through the potting tube and the bone shaft to provide additional fixation. The whole construct was mounted onto a material testing machine (Hounsfield H25K-S, Hounsfield Test Equipment., Salfords, Redhill, UK) with the two tunnels oriented along the direction of tensile load. The sutures fixing the tendon to bone tunnel were removed. To set the "zero" load state, a displacement was applied with a $50 \mathrm{~N}$ or $10 \mathrm{~N}$ load cell slowly until the loading force increased (about $0.02 \mathrm{~N}$ ), indicating that the construct was now under tension, and then the load cell was returned towards the initial position slightly. The construct was then loaded at a displacement rate of $20 \mathrm{~mm} / \mathrm{min}$ until failure after preloading at $0.5 \mathrm{~N}$ in moist condition. The sample was kept moist during the whole experiment. The ultimate load to failure $(\mathrm{N})$ and the failure mode were recorded. Stiffness $(\mathrm{N} / \mathrm{mm})$ of the healing complex was calculated from the linear portion of the load-displacement curve. Absolute values were compared as all the specimens have the same tunnel diameter and similar graft diameter.

\section{Histology}

The samples were fixed in $4 \%$ neutral buffered formalin, decalcified in $9 \%$ formic acid for about 4-6 weeks with the exact time determined by X-ray and embedded in paraffin as described previously (Lui et al., 2010b; Lui et al., 2011). The femoral and tibial tunnel complexes were divided into two, with the graft mid-substance preserved and attached to the tibial tunnel. $5 \mu \mathrm{m}$-thick longitudinal sections parallel to the direction of the bone tunnel were cut and stained with Haematoxylin and Eosin (H\&E) for the examination of healing at the tendon graft-bone tunnel interface under light microscopy. Sharpey's fibre was examined by polarised light microscopy (Leica DMRXA2, Leica Microsystems, Wetzlar, Germany). Only those sections where the entry and exit of bone tunnel could be identified were selected for histological assessment. One section was selected for histological assessment for each sample. The femoral tunnel, epiphyseal and metaphyseal regions of tibial tunnel were examined. Graft to bone tunnel healing was scored by one of the coinvestigators based on (a) tunnel graft integrity ( $\%$ of total graft area), (b) connection between tendon graft and bone (\% of graft perimeter) and (c) head-to-head connection between tendon graft and bone ( $\%$ of graft perimeter) at different tunnel regions. One slide from each sample was used for scoring. The median score was calculated and graded for each evaluation item of each group at each region using the following grading system: 0: $0 \%$; +: $0<\mathrm{x} \leq 20 \%$; ++: $20<\mathrm{x} \leq 40 \%$; +++: $40<\mathrm{x} \leq 60 \%$; ++++: $60<\mathrm{x} \leq 80 \%$; ++++++: $80<\mathrm{x} \leq 100 \%$

Higher grade indicated better tendon graft to bone tunnel healing. Representative sections having the median scores and matching the conclusion of image analysis were selected for presentation.

\section{Immunohistochemical staining}

The immunohistochemical staining of MMP1, MMP13 and CD68 (a monocyte-macrophage lineage marker that labels all potential osteoclast precursors and osteoclasts) was done as described previously and below (Lui et al. 2010a). Briefly, after removal of paraffin and rehydration, endogenous peroxidase activity was quenched with $3 \%$ hydrogen peroxide for $20 \mathrm{~min}$ at room temperature. Antigen retrieval was then performed with citrate buffer at $65{ }^{\circ} \mathrm{C}$ for $20 \mathrm{~min}$. After blocking with $5 \%$ normal goat serum, the sections were stained with specific antibodies against MMP1 (1:100; Novus Biologicals, Littleton, CO, USA), MMP13 (1:100; Abcam, Cambridge, UK) or CD68 $(1 ; 100 ;$ Abcam, Cambridge, UK) in a humid chamber at $4{ }^{\circ} \mathrm{C}$ overnight. The spatial and temporal localisation of these proteins were visualised by Lab Vision (Thermo Fisher Scientific., Waltham, MA, USA). Afterwards, the sections were rinsed, counterstained with haematoxylin, dehydrated with graded ethanol and xylene, and mounted with p-xylene-bis-pyridinium bromide (DPX) Permount (Sigma-Aldrich). Primary antibody was replaced with blocking solution in the controls. For good reproducibility and comparability, all the incubation times and conditions were strictly controlled. The sections were examined under light microscopy (Leica DMRXA2,). The femoral region, epiphyseal and metaphyseal regions of tibia of one slide from each sample were examined and graded as $0,+,++$, ,,++++++++++++ based on the signal intensity by the same co-investigator. The median grades at different graft to bone tunnel interface and peri-tunnel regions in each group, at week 6 after ACL reconstruction, were presented. Representative sections having the median grades and matching the conclusion of image analysis were selected for presentation.

\section{Data analysis}

VivaCT and biomechanical test data are shown in boxplots. Kruskal-Wallis test, followed by post-hoc pairwise comparison of different groups with Mann-Whitney U test, was used for comparing different groups at each time point. $p \leq 0.050$ was regarded as statistically significant.

\section{Results}

\section{Mineralised tissue formation inside bone tunnel}

There was a significant improvement in BMD and BV/ $\mathrm{TV}$ at the femoral tunnel and metaphyseal region of tibial tunnel at week 2 and week 6 after treatment with different doses of alendronate, except for BV/TV at the femoral 
Tunnel BMD

a

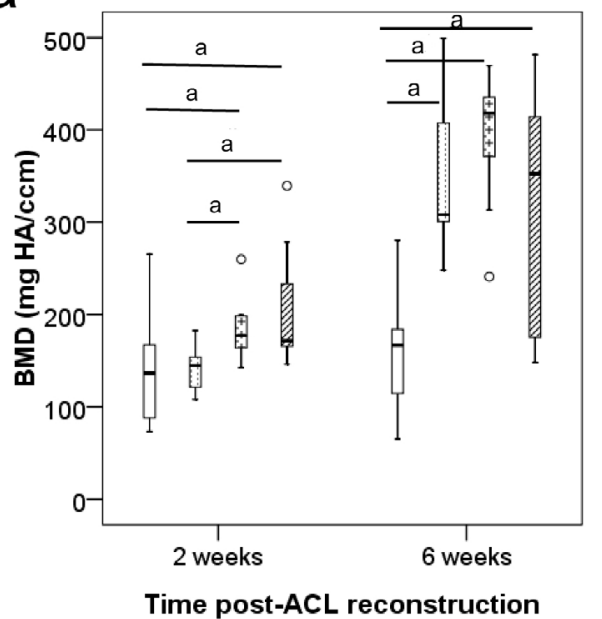

C

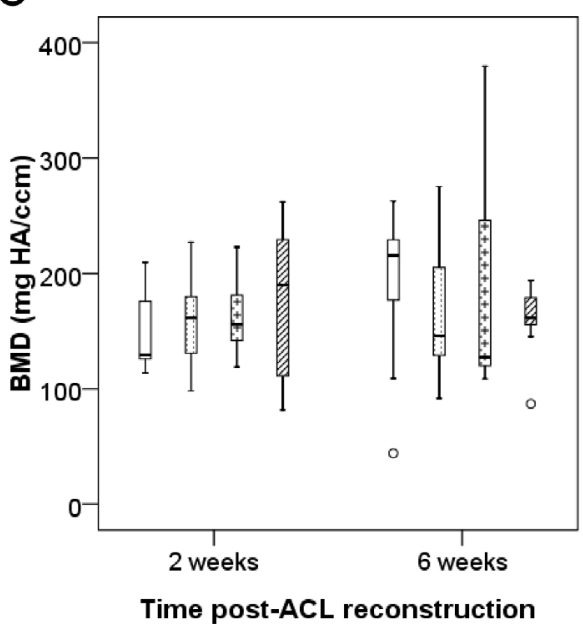

e

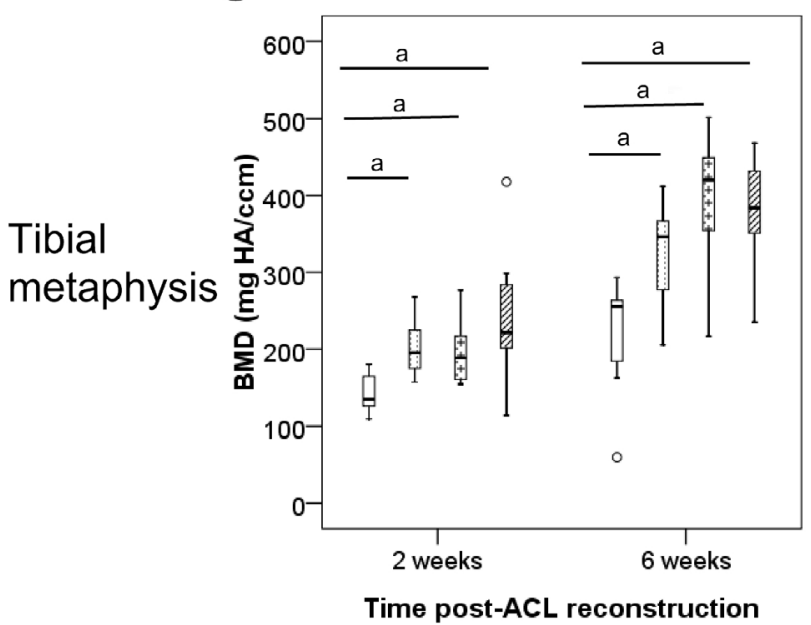

Tunnel BV/TV

b

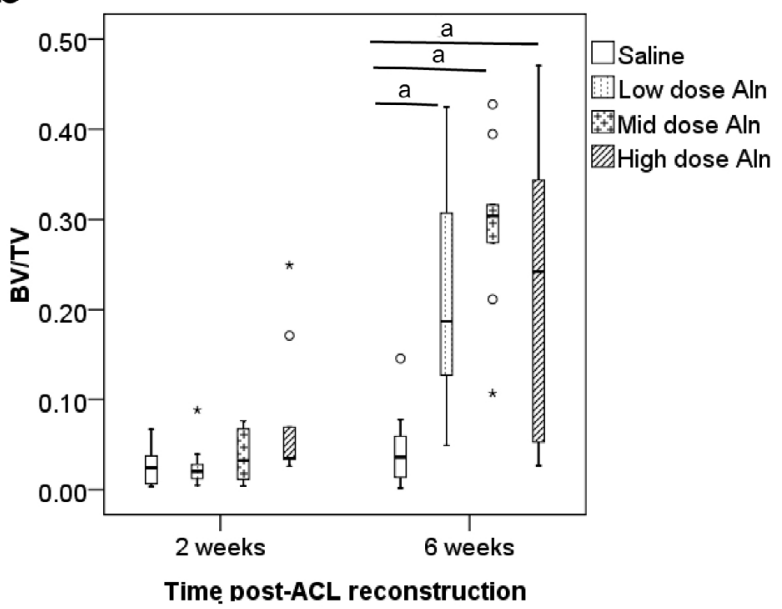

d

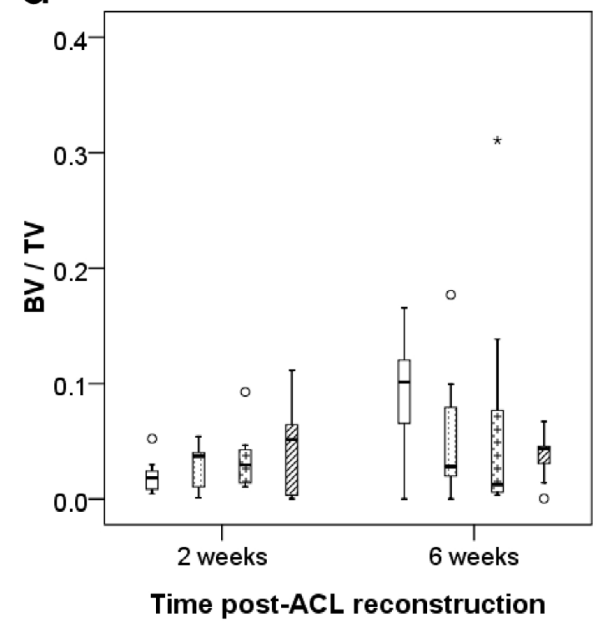

f

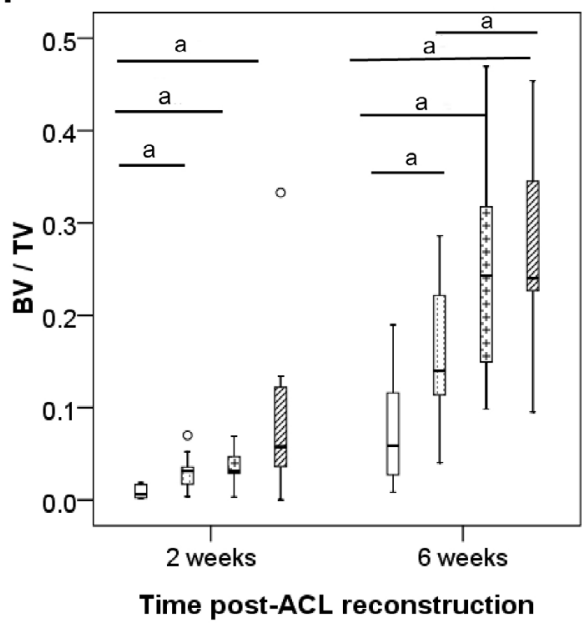

Fig. 2. Boxplots showing (a, c, e) BMD (mg HA/ccm) and (b, d, f) BV/TV of mineralised tissue inside the femoral tunnel $(\mathbf{a}, \mathbf{b})$, epiphyseal $(\mathbf{c}, \mathbf{d})$ and metaphyseal $(\mathbf{e}, \mathbf{f})$ regions of tibial tunnel in different treatment groups at week 2 and week 6. a: $p \leq 0.050$ in post-hoc comparison. 


\section{Alendronate Saline Low Mid High}

$\stackrel{0}{\frac{0}{0}}$
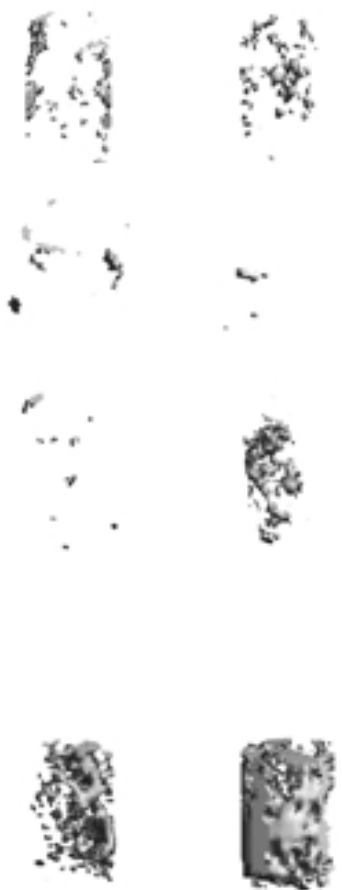

0
0
0
0
0
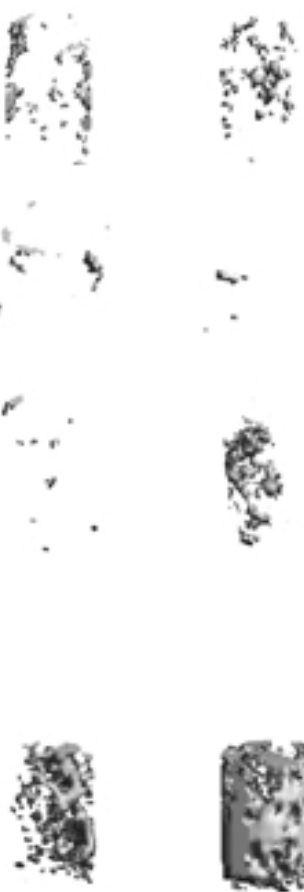

$\hookrightarrow$
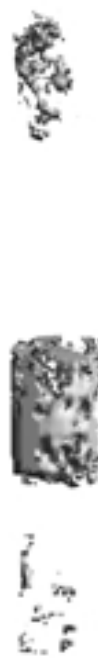

$-1$

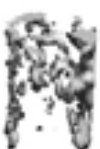

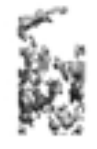
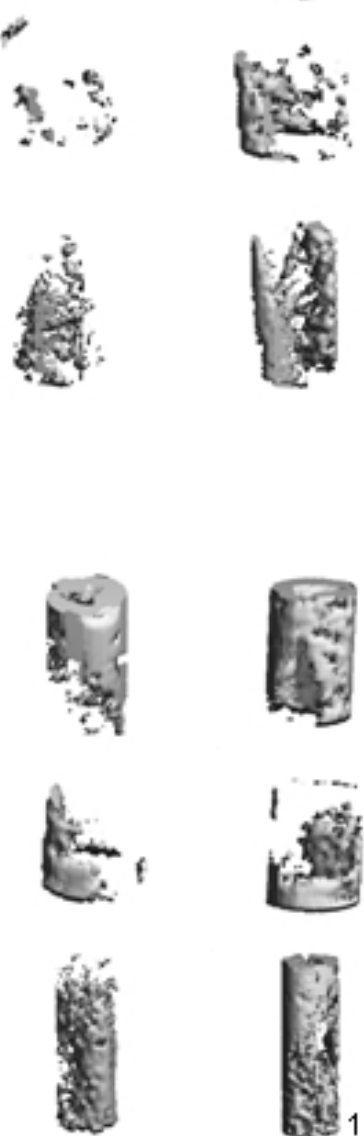

Femoral

Tibial

epiphysis

Tibial

metaphysis
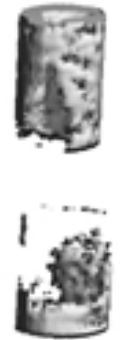

Femoral

Tibial epiphysis

\section{Tibial}

metaphysis

Fig. 3. Representative vivaCT images showing the mineralised tissue inside different tunnel regions in different treatment groups at week 2 and week 6 . Scale bar $=1 \mathrm{~mm}$.

tunnel at week 2 (Fig. 2a-b, e-f). There was no significant difference in BMD and BV/TV at the epiphyseal region of tibial tunnel among different groups at both week 2 and week 6 (Fig. 2 c-d). Dose-dependent effect of alendronate on the formation of mineralised tissue inside the bone tunnel was observed at the femoral tunnel where significantly higher BMD was observed in the high-dose (204 $\pm 67 \mathrm{mg} \mathrm{HA} / \mathrm{ccm})$ and mid-dose $(183 \pm 36 \mathrm{mg} \mathrm{HA} /$ $\mathrm{ccm})$ alendronate groups compared to that in the lowdose group (142 $\pm 27 \mathrm{mg} \mathrm{HA} / \mathrm{ccm}$ ) at week 2 (Fig. 2a). There was also significantly higher BV/TV with highdose $(0.28 \pm 0.11)$ compared to low-dose $(0.16 \pm 0.08)$ alendronate treatment at the metaphyseal region of tibial tunnel at week 6 (Fig. 2f). Fig. 3 shows the representative vivaCT images of the mineralised tissue inside bone tunnels in different groups.

\section{Bone tunnel diameter changes}

VivaCT analysis revealed no significant change in tunnel diameter among different groups except at the femoral tunnel at week 6 (overall $p=0.039$ ) (Fig. 4). The tunnel diameter was significantly smaller in the mid-dose $(0.55 \pm 0.27 \mathrm{~mm})$ and high-dose alendronate $(0.62 \pm 0.17 \mathrm{~mm})$ groups compared to that in the saline group $(0.81 \pm 0.19 \mathrm{~mm})$ at week 6 (Fig. $4 b)$.

\section{Peri-tunnel bone changes}

The peri-tunnel bone density and mass decreased from week 2 to week 6 in the saline group and was statistically significant at the tibial epiphysis (peri-tunnel BMD: $483.5 \pm 70.3 \mathrm{mg} \mathrm{HA} / \mathrm{ccm}$ to $275.3 \pm 41.7 \mathrm{mg} \mathrm{HA} / \mathrm{ccm}$; peri-tunnel BV/TV: $0.385 \pm 0.110$ to $0.142 \pm 0.050$; both $p<0.001$ ) (Fig. $5 \mathrm{c}-\mathrm{d}$ ) and metaphysis (peri-tunnel BMD: $127.5 \pm 37.0 \mathrm{mg} \mathrm{HA} / \mathrm{ccm}$ to $47.0 \pm 32.3 \mathrm{mg} \mathrm{HA} / \mathrm{ccm}$; peri- 
a

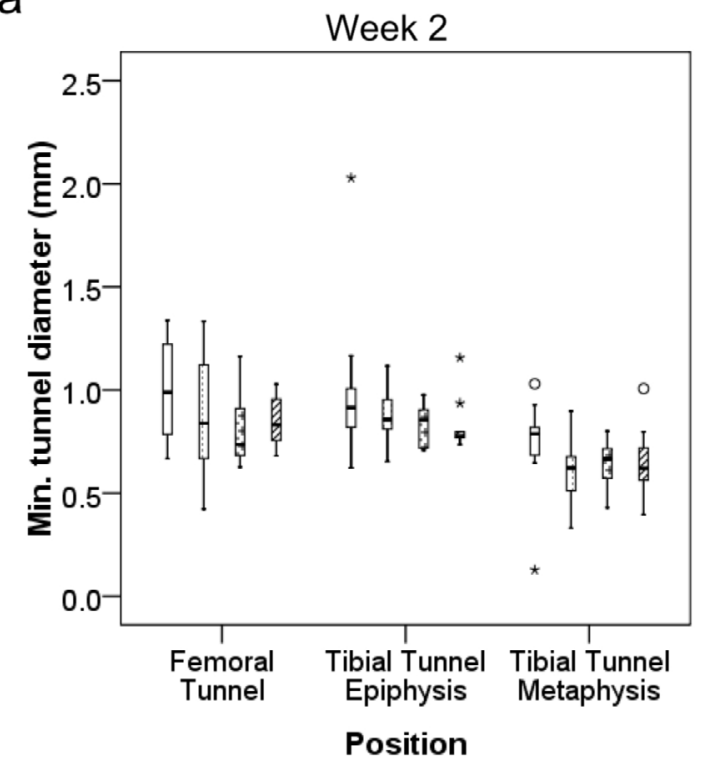

b

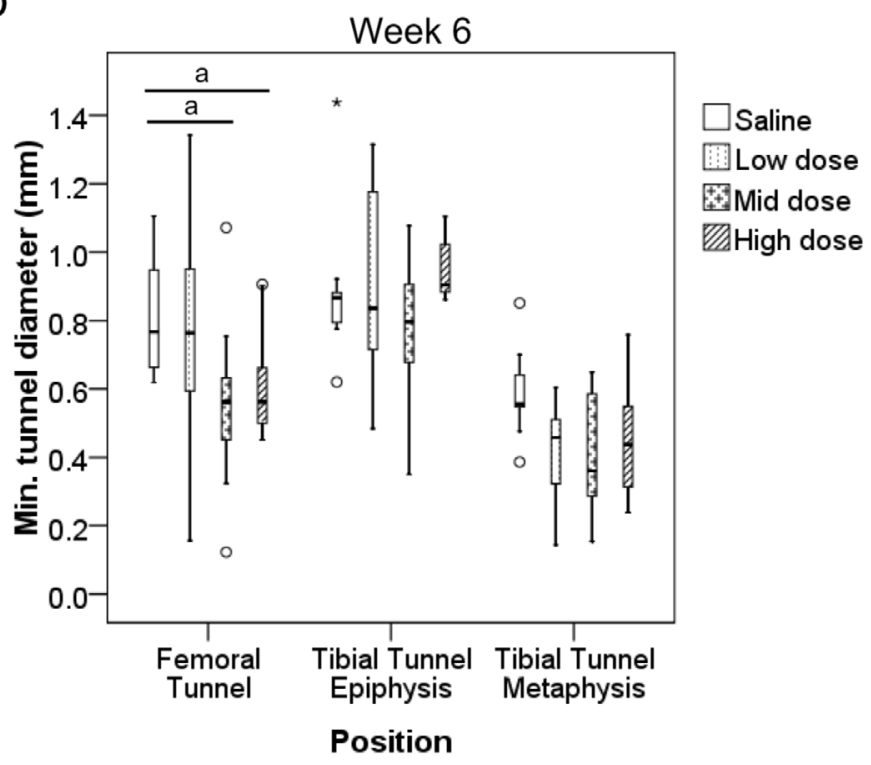

Fig. 4. Boxplots showing the tunnel diameter at the femoral tunnel, epiphyseal and metaphyseal regions of the tibial tunnel in different treatment groups at (a) week 2 and (b) week 6. a: $p \leq 0.050$ in post-hoc comparison.

Table 1. Histological grading of the tendon graft to bone tunnel healing at the femoral tunnel, tibial tunnel epiphysis and tibial tunnel metaphysis in different treatment groups at week 6.

\begin{tabular}{|c|c|c|c|}
\hline Group & Graft integrity (x) & $\begin{array}{c}\text { Connection between tendon } \\
\text { graft and bone (x) } \\
\end{array}$ & Head-to-head connection $(x)$ \\
\hline \multicolumn{4}{|l|}{ Femoral tunnel } \\
\hline Saline & 0 & +++++ & + \\
\hline Low-dose alendronate & +++ & +++++ & ++++ \\
\hline Mid-dose alendronate & +++ & +++++ & ++ \\
\hline High-dose alendronate & +++ & +++++ & ++++ \\
\hline \multicolumn{4}{|l|}{ Tibial tunnel epiphysis } \\
\hline Saline & + & +++ & + \\
\hline Low-dose alendronate & +++ & +++++ & ++ \\
\hline Mid-dose alendronate & ++++ & +++++ & +++ \\
\hline High-dose alendronate & ++ & +++++ & ++ \\
\hline \multicolumn{4}{|c|}{ Tibial tunnel metaphysis } \\
\hline Saline & + & +++ & + \\
\hline Low-dose alendronate & ++++ & +++++ & +++ \\
\hline Mid-dose alendronate & +++++ & +++++ & ++++ \\
\hline High-dose alendronate & ++++ & +++++ & +++ \\
\hline
\end{tabular}

tunnel BV/TV: $0.009 \pm 0.008$ to $0.003 \pm 0.009 ; p=0.001$, 0.005, respectively) (Fig. 5 e-f).

At week 2, there was significant improvement in peritunnel BMD at the femoral epiphysis in the high-dose alendronate group $(513 \pm 90 \mathrm{mg} \mathrm{HA} / \mathrm{ccm})$ compared to the saline group $(380 \pm 101 \mathrm{mg} \mathrm{HA} / \mathrm{ccm})$ (Fig. 5a). At week 2, there was also significant higher peri-tunnel BMD (Fig. 5e) and BV/TV (Fig. 5f) at the tibial metaphysis at all tested doses of alendronate compared to the saline group. At week 6, the peri-tunnel BMD and BV/TV in the alendronate groups were significantly higher than those in the saline group, at all regions along both the femoral and tibial tunnels (Fig. 5a-f). There was dose-dependent increase in peri-tunnel BMD and BV/TV only at the tibial metaphysis (Fig. 5e-f). The peri-tunnel BV/TV and BMD at the femoral epiphysis and tibial epiphysis in the highdose alendronate group were reduced compared to those in the mid-dose alendronate group (Fig. 5a-d). The vivaCT data was also confirmed by histology which showed more bone next to the bone tunnel in the alendronate-treated groups compared to that in the control group at the tibial metaphysis close to the growth plate at both week 2 and 
Peri-tunnel BMD

a

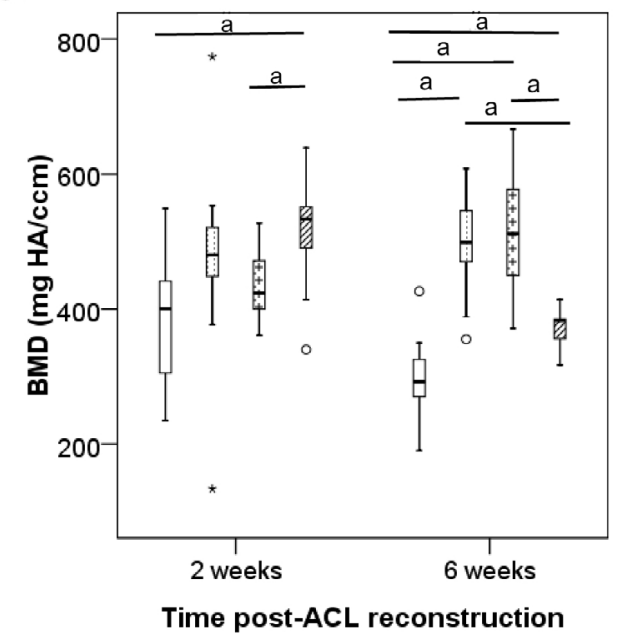

C

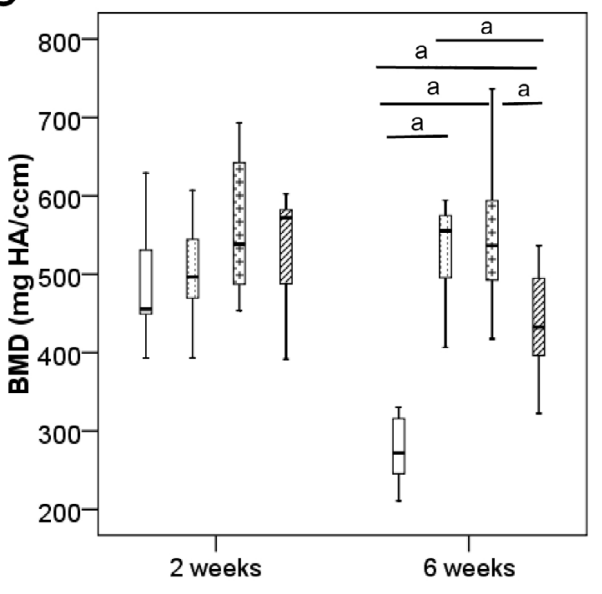

e

Time post-ACL reconstruction

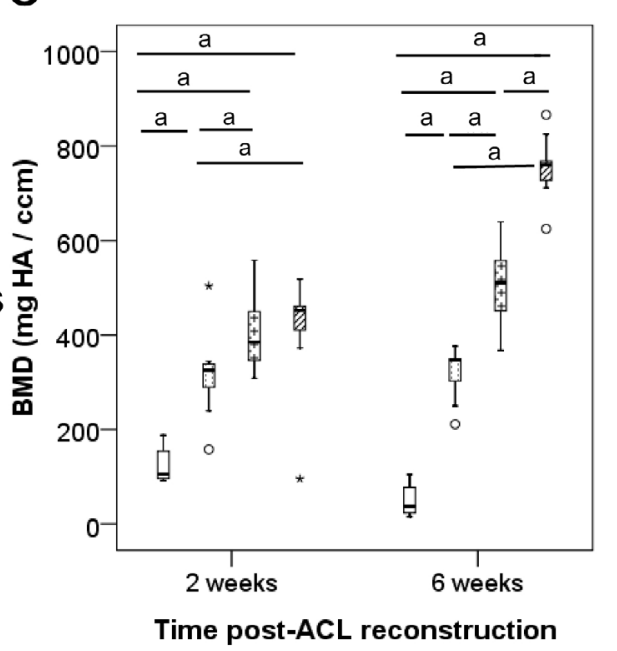

Peri-tunnel BV/TV

b

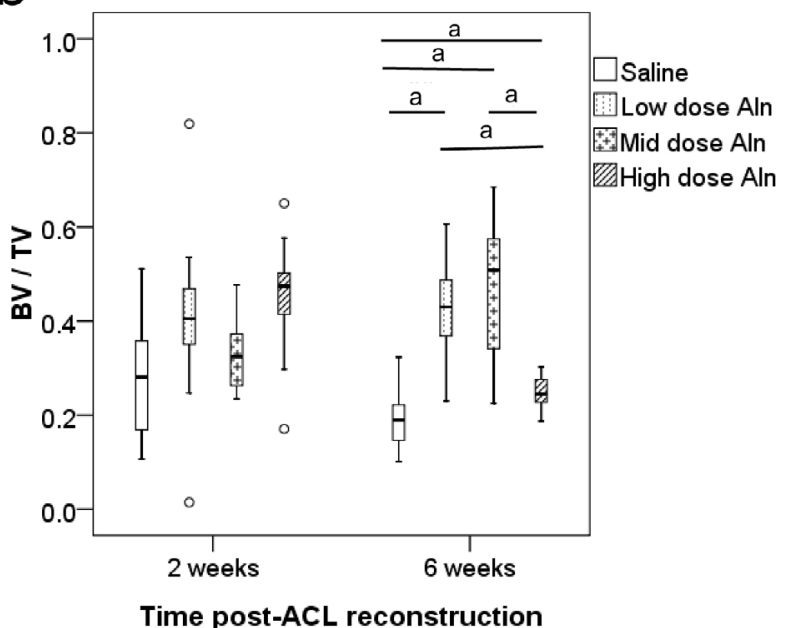

d

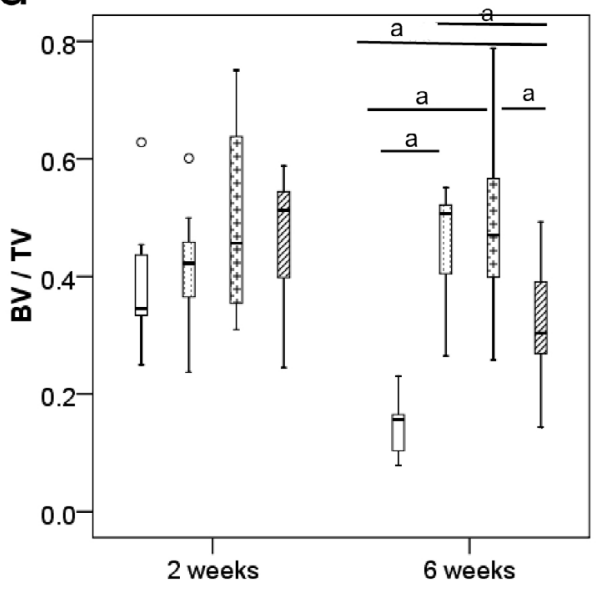

Time post-ACL reconstruction

f

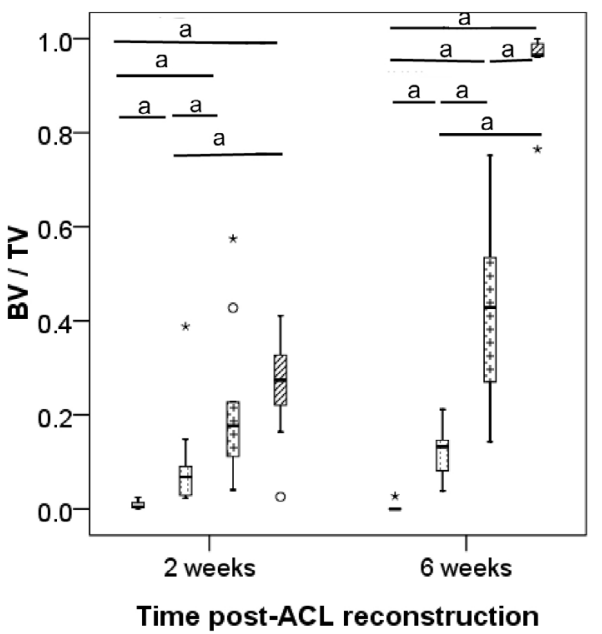

Fig. 5. Boxplots showing (a, c, e) BMD (mg HA/ccm) and (b, d, f) BV/TV of peri-tunnel bone at the (a, b) femoral epiphysis, $(\mathbf{c}, \mathbf{d})$ tibial epiphysis and $(\mathbf{e}, \mathbf{f})$ tibial metaphysis in different treatment groups at week 2 and week 6 . a: $p \leq 0.050$ in post-hoc comparison. 


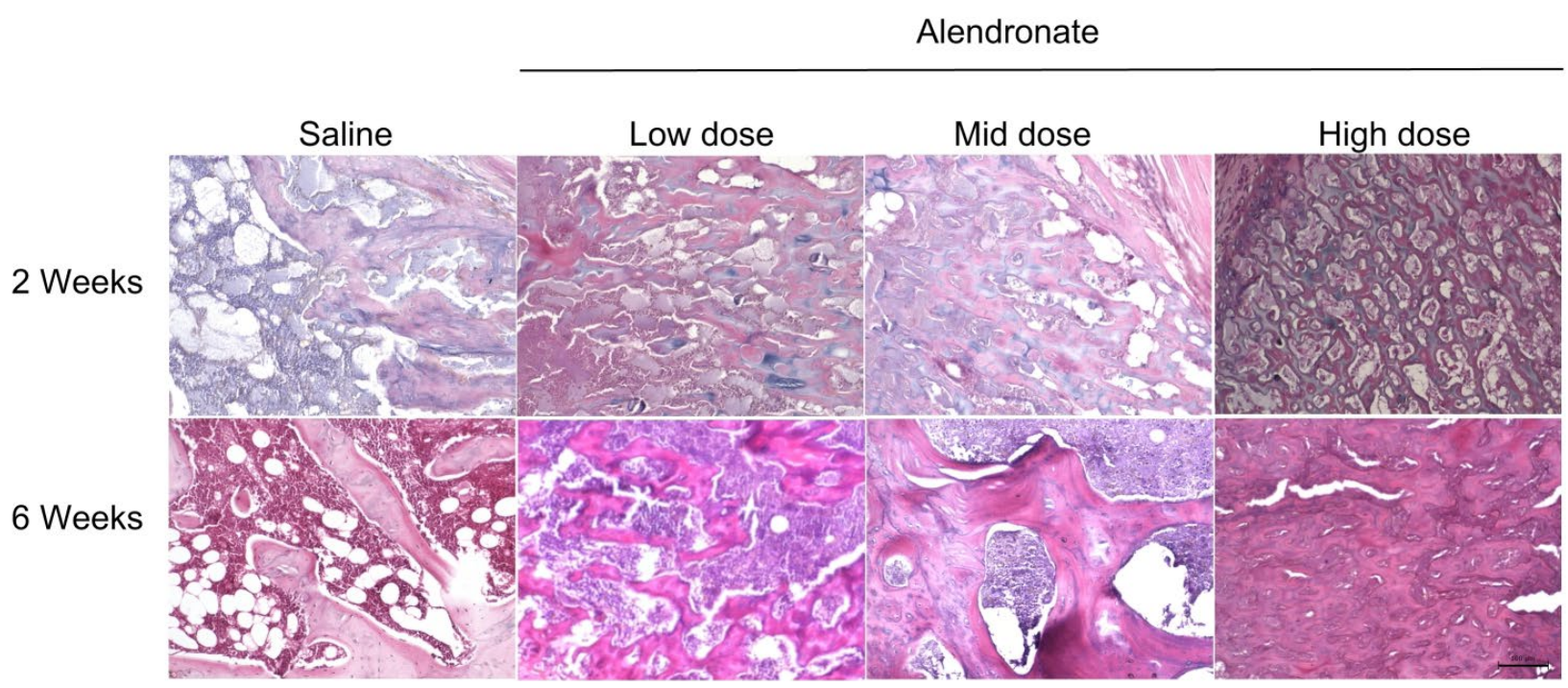

Fig. 6. Representative photomicrographs showing peri-tunnel bone mass at the tibial metaphysis in different treatment groups at week 2 and week 6 . Note the higher bone mass in the alendronate groups compared to that in the saline group and the effect was dose-dependent. H\&E staining; Scale bar $=100 \mu \mathrm{m}$.

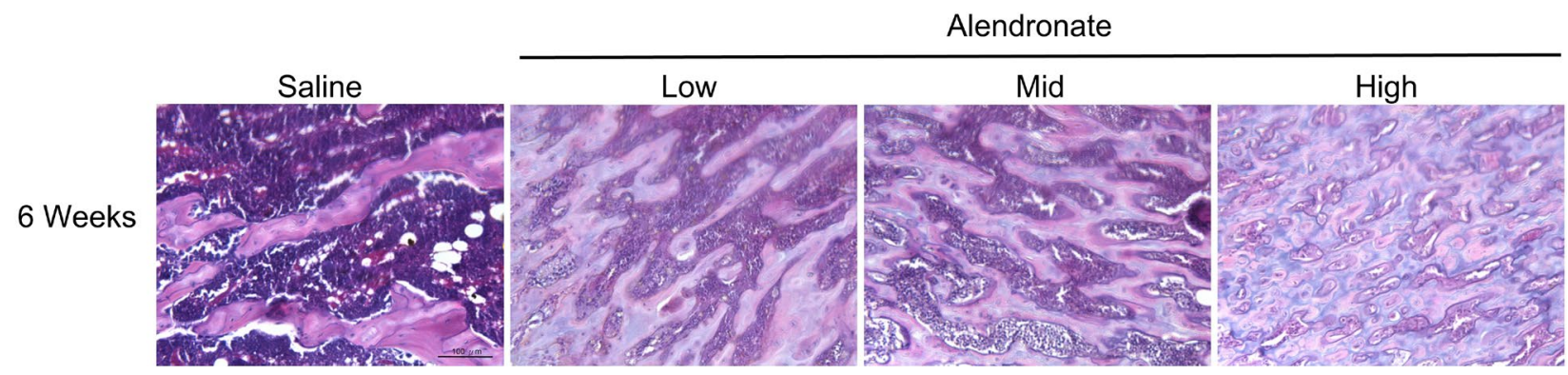

Fig. 7. Representative photomicrographs showing peri-tunnel bone mass at the tibial metaphysis in different treatment groups at week 6. H\&E staining; Scale bar $=100 \mu \mathrm{m}$. (Fig. 7, 9 and $\mathbf{1 1}$ show similar findings to Figs. 6, 8 and 10, respectively, at week 6 using intact graft-bone complex for histology. Similar findings were observed, suggesting the validity of the histological observations using the graft-bone complex after biomechanical test).

Table 2. Failure mode of bone-tendon graft-bone complex during biomechanical testing.

\begin{tabular}{clccc}
\hline $\begin{array}{c}\text { Time } \\
\text { post-ACL } \\
\text { reconstruction }\end{array}$ & \multicolumn{1}{c}{ Treatment groups } & $\begin{array}{c}\text { Femoral tunnel } \\
\text { pull-out }\end{array}$ & $\begin{array}{c}\text { Tibial tunnel } \\
\text { pull-out }\end{array}$ & $\begin{array}{c}\text { Mid-substance } \\
\text { failure }\end{array}$ \\
\hline \multirow{5}{*}{ Week 2 } & Saline & 9 & 0 & 0 \\
& Low-dose alendronate $(6 \mu \mathrm{g} / \mathrm{kg} / \mathrm{week})$ & 9 & 0 & 0 \\
& Mid-dose alendronate $(60 \mu \mathrm{g} / \mathrm{kg} / \mathrm{week})$ & 9 & 0 & 0 \\
& High-dose alendronate $(300 \mu \mathrm{g} / \mathrm{kg} / \mathrm{week})$ & 9 & 0 & 0 \\
\hline \multirow{5}{*}{ Week 6 } & Saline & 0 & 0 & 9 \\
& Low-dose alendronate $(6 \mu \mathrm{g} / \mathrm{kg} / \mathrm{week})$ & 0 & 0 & 9 \\
& Mid-dose alendronate $(60 \mu \mathrm{g} / \mathrm{kg} / \mathrm{week})$ & 0 & 0 & 9 \\
\hline
\end{tabular}

week 6 (Fig. 6). Dose-dependent effect of alendronate on peri-tunnel bone at the tibial metaphysis was observed by histology at week 6 at the tibial metaphysis (Fig. 6). Similar histological findings were observed at the peri-tunnel area of graft-bone complex harvested at week 6 without prior biomechanical test (Fig. 7).

Graft-bone tunnel integration and graft midsubstance remodelling

Histological analysis demonstrated an improvement in tendon graft to bone tunnel integration and tunnel graft integrity after alendronate treatment. At week 6, the tendon graft in the saline group has high cellularity and the collagen fibre was either degraded or loose along both tunnels, resulting in loose connection between tendon graft and bone at the interface (Table 1, Fig. 8 a, e, i). On the other hand, the tendon graft along both bone tunnels showed higher integrity and low cellularity in the alendronate groups (Table 1, Fig. 8). More Sharpey's fibres were observed along the femoral tunnel and tibial tunnel in the alendronate groups, compared with that in the saline group (Table 1, Fig. 8). Similar histological findings were 

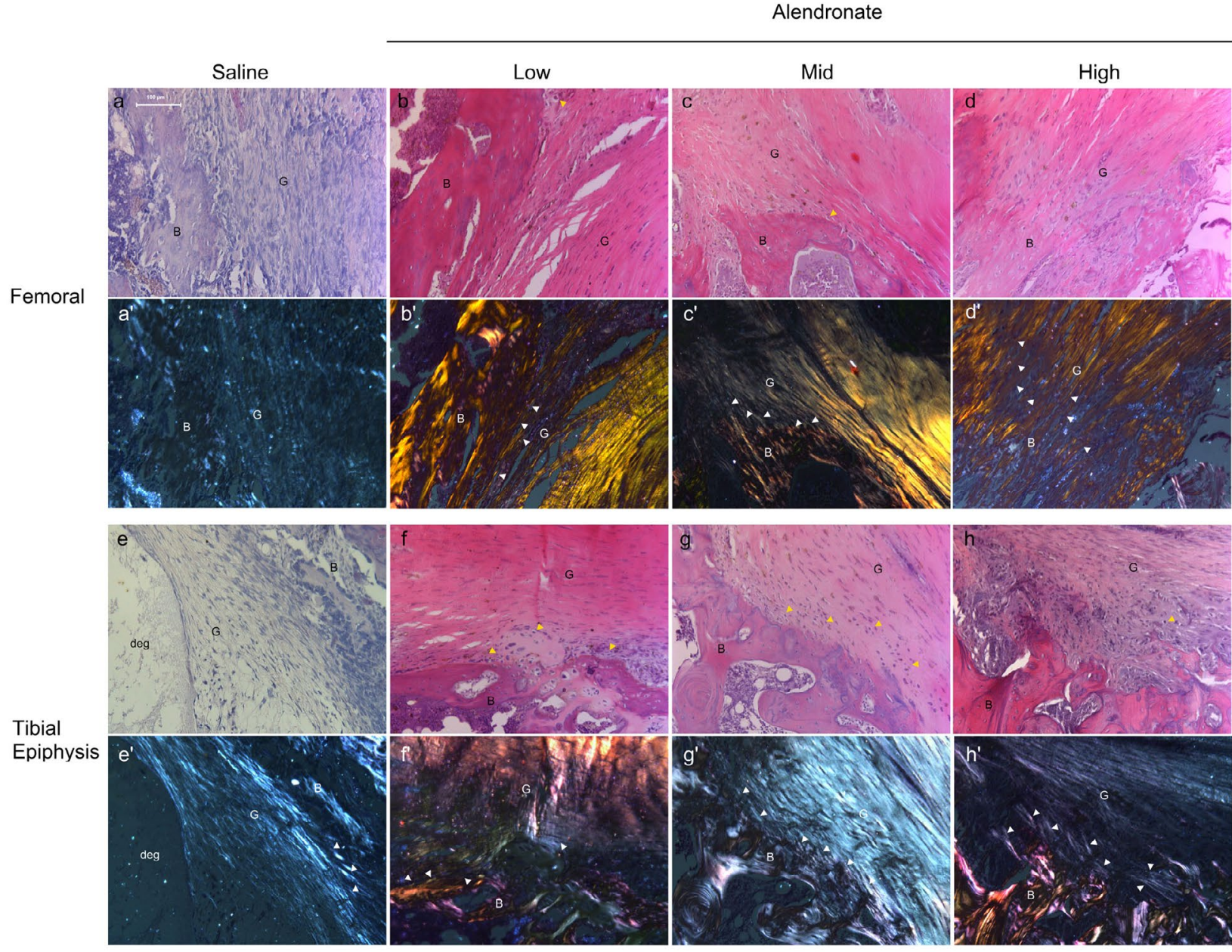

Tibial
Epiphysis
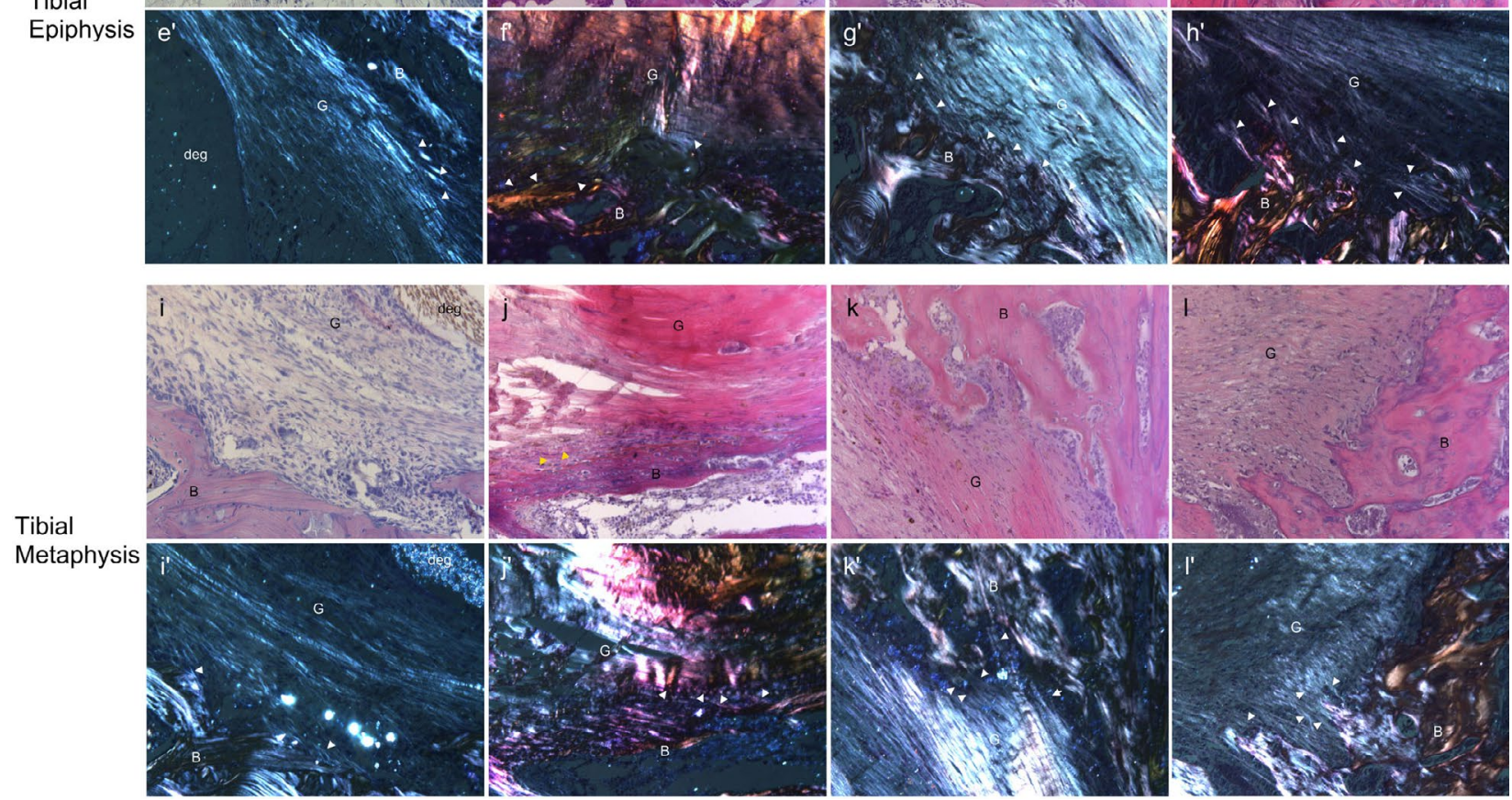

Fig. 8. Representative photomicrographs showing tendon graft to bone tunnel healing at different tunnel regions in the saline and alendronate groups at week 6. Panels (a-l) show the H\&E staining while panels (a'-l') show the polarised images of the same view. H\&E staining; Scale bar $=100 \mu \mathrm{m}$; B: bone; G: graft; deg: degenerated graft; white arrowhead: Sharpey's fibres; yellow arrowhead: chondrocyte-like cells.

observed for the samples harvested at week 6 without prior biomechanical test (Fig. 9).

The graft mid-substance showed high cellularity with loose collagen fibres and there was no difference among different groups at both week 2 and week 6 (Fig. 10). Similar results were observed at the graft mid-substance at week 6 for samples without prior biomechanical test (Fig. 11).

\section{Biomechanical test}

There was significant higher ultimate load in the mid-dose $(6.7 \pm 1.7 \mathrm{~N})$ and high-dose $(6.1 \pm 1.1 \mathrm{~N})$ alendronate groups compared to that in the saline group $(2.8 \pm 1.9 \mathrm{~N})$ at week 2 (Fig. 12a). The ultimate loads in the high-dose and mid-dose alendronate groups were higher than that in the low-dose group $(4.5 \pm 2.1 \mathrm{~N})$ at week 2 in the posthoc analysis (Fig. 12a). The stiffness at week 2 increased with alendronate treatment but this increase was not 
Table 3. Histological grading of immunohistochemical staining of CD68, MMP1 and MMP13 in the saline group and mid-dose alendronate group at week 6 after ACL reconstruction.

\begin{tabular}{|c|c|c|c|}
\hline & Group & Peri-tunnel region & Graft-bone tunnel interface \\
\hline \multicolumn{4}{|l|}{ CD68 } \\
\hline \multicolumn{4}{|c|}{ Femur (week 6) } \\
\hline & Saline & ++++ & +++ \\
\hline & Mid-dose alendronate & + & ++ \\
\hline \multicolumn{4}{|c|}{ Tibial epiphysis (week 6) } \\
\hline & Saline & +++++ & +++++ \\
\hline & Mid-dose alendronate & + & + \\
\hline \multicolumn{4}{|c|}{ Tibial metaphysis (week 6) } \\
\hline & Saline & +++++ & +++++ \\
\hline & Mid-dose alendronate & ++ & ++ \\
\hline \multicolumn{4}{|l|}{ MMP1 } \\
\hline \multicolumn{4}{|c|}{ Femur (week 6) } \\
\hline & Saline & +++ & +++ \\
\hline & Mid-dose alendronate & ++ & + \\
\hline \multicolumn{4}{|c|}{ Tibial epiphysis (week 6) } \\
\hline & Saline & +++ & +++++ \\
\hline & Mid-dose alendronate & + & ++ \\
\hline \multicolumn{4}{|c|}{ Tibial metaphysis (week 6) } \\
\hline & Saline & +++++ & ++++ \\
\hline & Mid-dose alendronate & + & ++ \\
\hline \multicolumn{4}{|l|}{ MMP13 } \\
\hline \multicolumn{4}{|c|}{ Femur (week 6) } \\
\hline & Saline & +++++ & +++++ \\
\hline & Mid-dose alendronate & +++ & +++ \\
\hline \multicolumn{4}{|c|}{ Tibial epiphysis (week 6) } \\
\hline & Saline & +++++ & +++++ \\
\hline & Mid-dose alendronate & ++ & ++ \\
\hline \multicolumn{4}{|c|}{ Tibial metaphysis (week 6) } \\
\hline & Saline & +++++ & +++++ \\
\hline & Mid-dose alendronate & + & ++ \\
\hline
\end{tabular}

The samples were graded according the signal intensity as $0,+,++,+++,++++$ and +++++ . The median grades at different tunnel and peri-tunnel regions in each group at week 6 after ACL reconstruction are shown.

statistically significant (Fig. 12b). No significant difference in the ultimate load (saline, low-dose, mid-dose, highdose alendronate: $6.5 \pm 2.5 \mathrm{~N}$ versus $10.6 \pm 4.5 \mathrm{~N}$ versus $7.8 \pm 5.9 \mathrm{~N}$ versus $8.5 \pm 3.9 \mathrm{~N}$ ) and stiffness (saline, lowdose, mid-dose, high-dose alendronate: $11.2 \pm 4.0 \mathrm{~N} / \mathrm{mm}$ versus $12.1 \pm 4.8 \mathrm{~N} / \mathrm{mm}$ versus $11.6 \pm 9.4 \mathrm{~N} / \mathrm{mm}$ versus $13.7 \pm 6.3 \mathrm{~N} / \mathrm{mm}$ ) of the bone-graft-bone complex was observed among different groups at week 6 (Fig. 12). All samples failed by graft pull-out from the femoral tunnel at week 2 and graft mid-substance failure at week 6 (Table 2).

\section{Immunohistochemical staining of MMP1, MMP13 and CD68}

There was lower expression of CD68+ cells, MMP1 and MMP13 at the graft to bone tunnel interface and the peritunnel bone in the mid-dose alendronate group compared to similar regions in the saline group at week 6 at all tunnel segments (Table 3, Figs. 13-15).

\section{Discussion}

Our results showed that alendronate significantly increased bone mass and density of mineralised tissue inside bone tunnel as well as reduced bone tunnel diameter. Better tendon graft to bone tunnel integration, with higher tunnel graft integrity and more Sharpey's fibres connecting between tendon graft and bone tunnel, were also observed in the alendronate groups. We observed similar histological findings for samples with and without prior biomechanical test at week 6 , suggesting that the histological observation based on samples with prior biomechanical test was valid. 


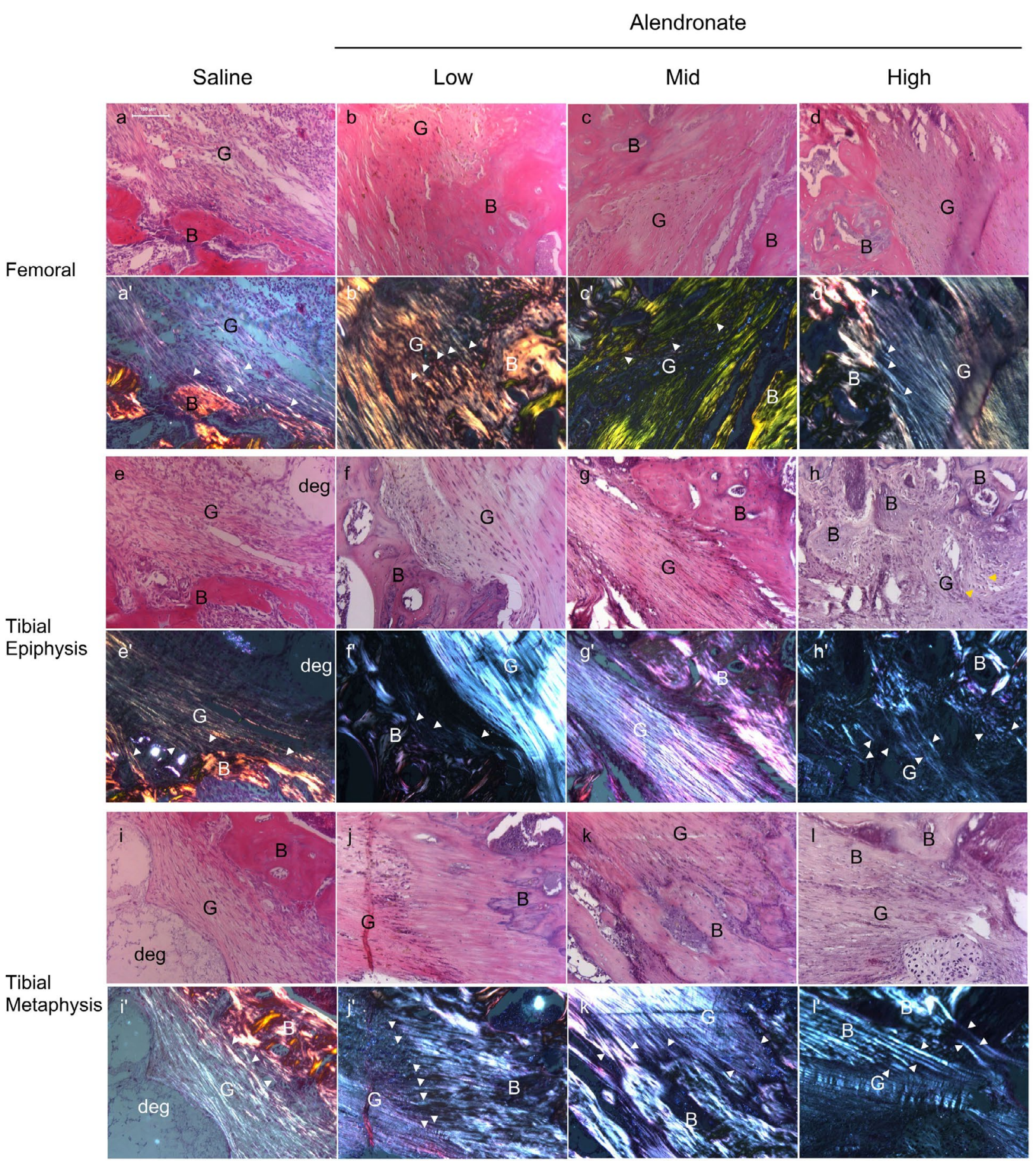

Fig. 9. Representative photomicrographs showing tendon graft to bone tunnel healing at different tunnel regions in the saline and alendronate groups at week 6. Panels (a-l) show the H\&E staining while panels (a'-l') show the polarised images of the same view. H\&E staining. Scale bar $=100 \mu \mathrm{m}$; B: bone; G: graft; deg: degenerated graft; white arrowhead: Sharpey's fibres; yellow arrowhead: chondrocyte-like cells.

Peri-tunnel bone loss was observed in this animal model. Treatment of rats with alendronate also increased the peritunnel bone density and mass; and hence reduced peritunnel bone loss after ACL reconstruction. The increase in peri-tunnel bone by alendronate was also confirmed by histology. The ultimate load was significantly higher in the alendronate groups compared with that in the control group at week 2. The improvement in stiffness at week 2 after alendronate treatment was not statistically significant.
The insignificant effect of alendronate on ultimate load and stiffness at week 6 might be due to the shift of the weak link from tendon graft-bone tunnel interface at week 2 to graft mid-substance at week 6 . Our results showed no difference in graft mid-substance remodelling among different groups at both week 2 and week 6 . However, the tunnel graft showed higher integrity and lower cellularity in the alendronate groups compared with the tunnel graft in the saline group. There was a trend of dose-dependent 


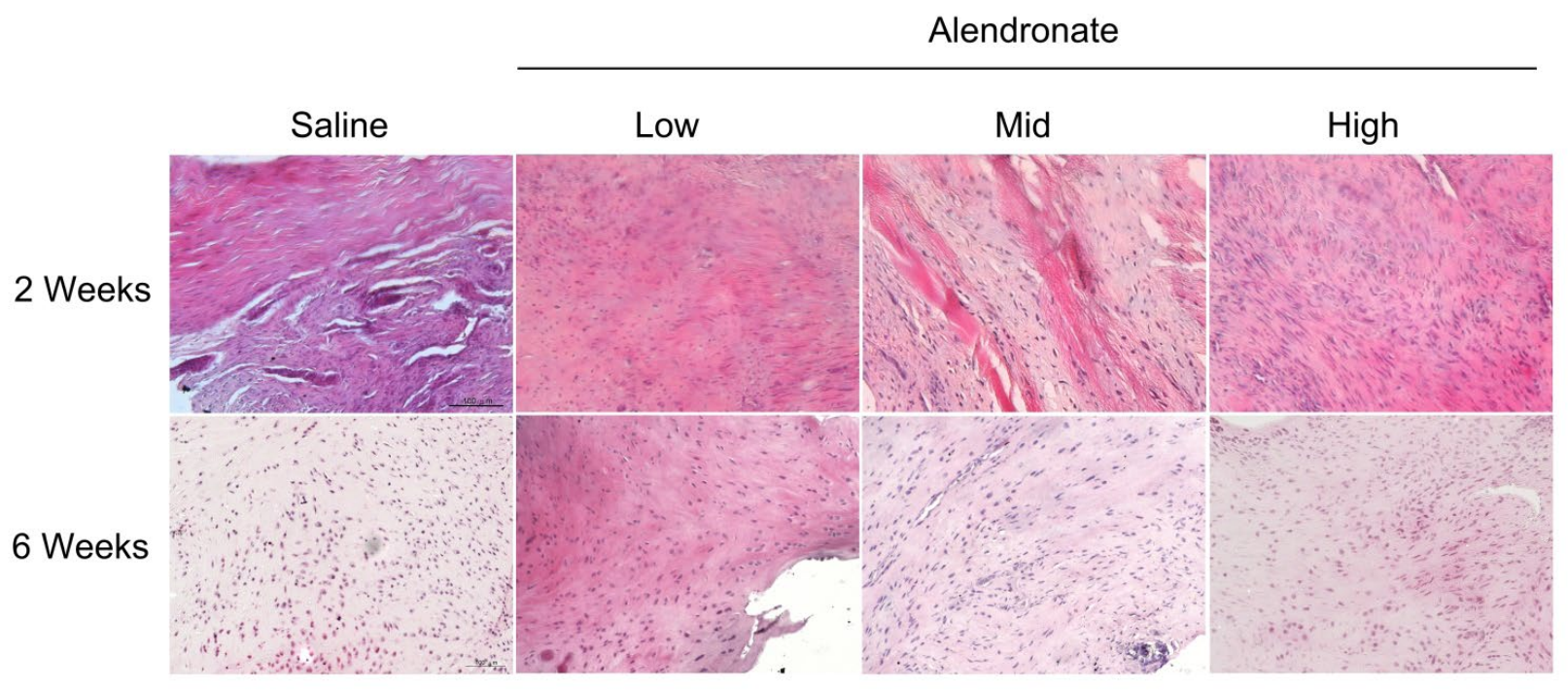

Fig. 10. Representative photomicrographs showing graft mid-substance remodelling in the saline, low-dose $(6 \mu \mathrm{g} /$ $\mathrm{kg} /$ week), mid-dose ( $60 \mu \mathrm{g} / \mathrm{kg} / \mathrm{week})$ and high-dose ( $300 \mu \mathrm{g} / \mathrm{kg} / \mathrm{week})$ alendronate groups at week 2 and week 6 . H\&E staining. Scale bar $=100 \mu \mathrm{m}$.

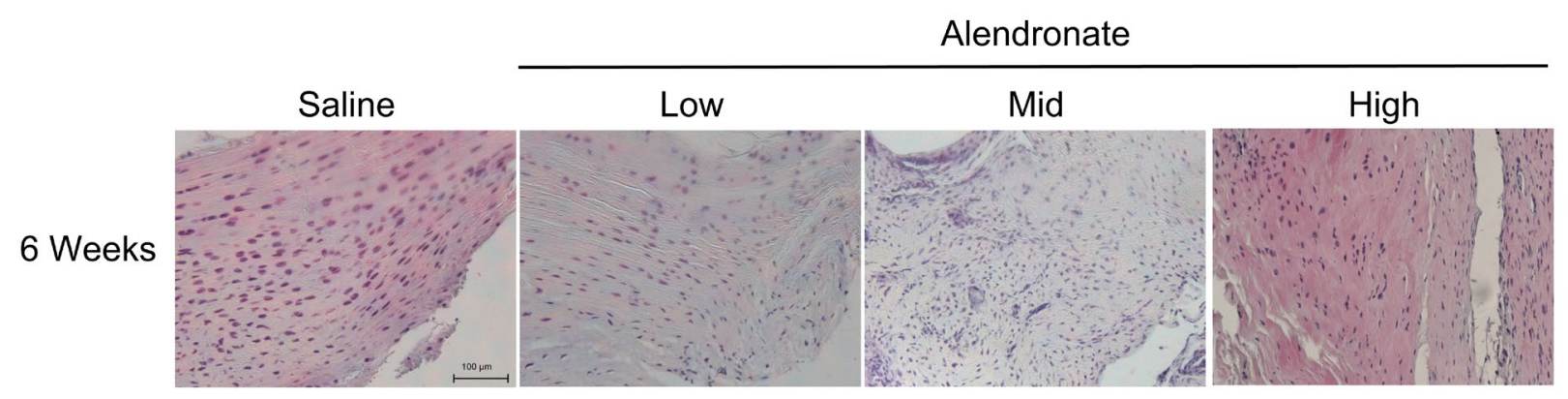

Fig. 11. Representative photomicrographs showing graft mid-substance remodelling in the saline, low-dose $(6 \mu \mathrm{g} /$ $\mathrm{kg} /$ week $)$, mid-dose $(60 \mu \mathrm{g} / \mathrm{kg} /$ week $)$ and high-dose $(300 \mu \mathrm{g} / \mathrm{kg} /$ week $)$ alendronate groups at week 6 . H\&E staining. Scale bar $=100 \mu \mathrm{m}$.

a

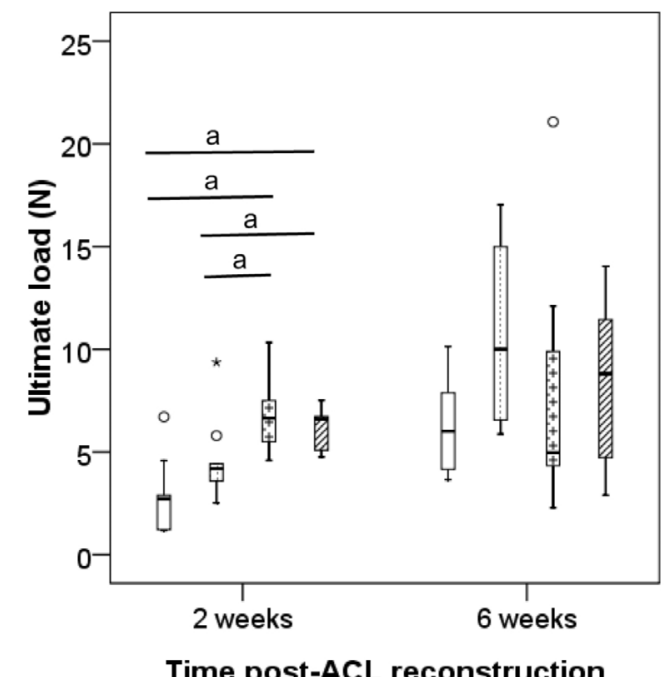

b

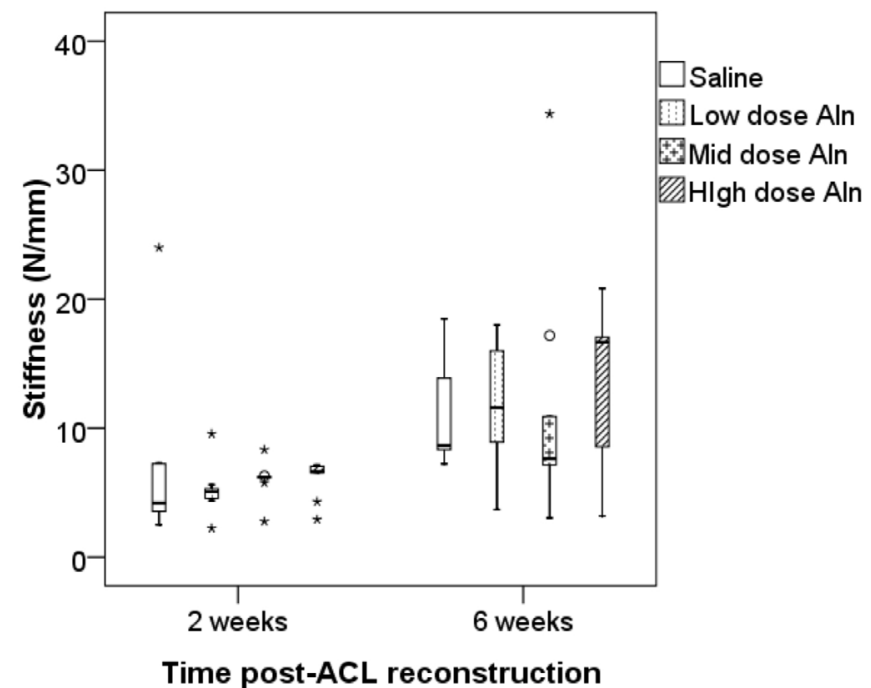

Fig. 12. Boxplots showing the (a) ultimate load $(\mathrm{N})$ and $(\mathbf{b})$ stiffness $(\mathrm{N} / \mathrm{mm})$ of the bone-tendon graft-bone complex at week 2 and week 6 in different treatment groups. a: $p \leq 0.050$ in post-hoc comparison. 


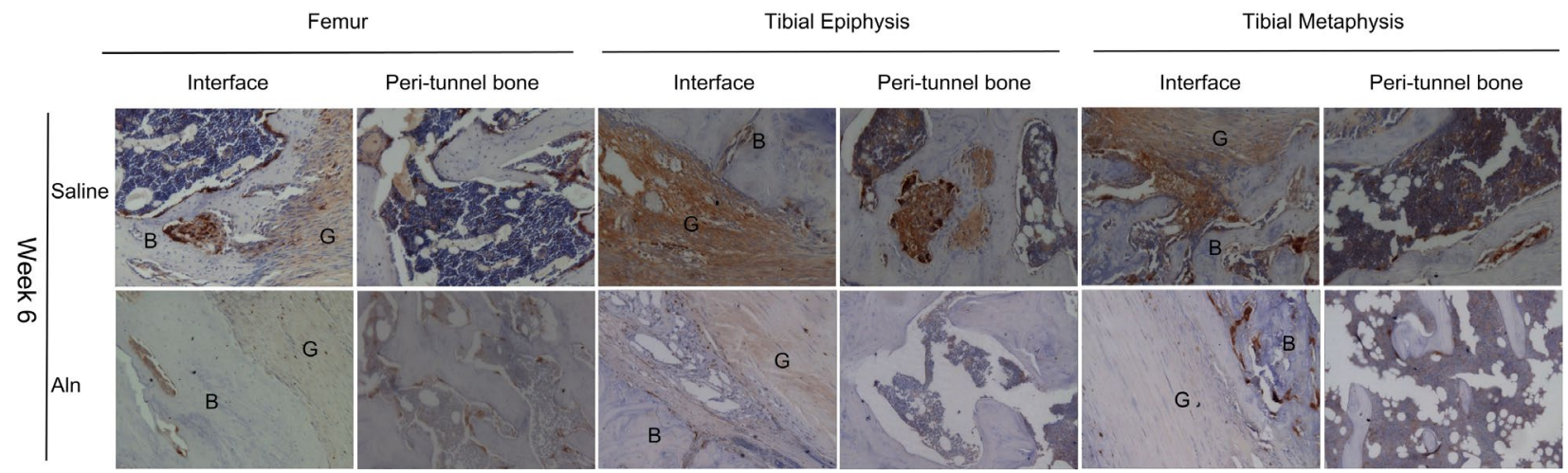

Fig. 13. Representative photomicrographs showing the immunohistochemical staining of CD68+ cells at different tunnel and peri-tunnel regions in the mid-dose alendronate group and saline group at week 6 after ACL reconstruction. Scale bar $=100 \mu \mathrm{m}$. B: bone; G: graft.

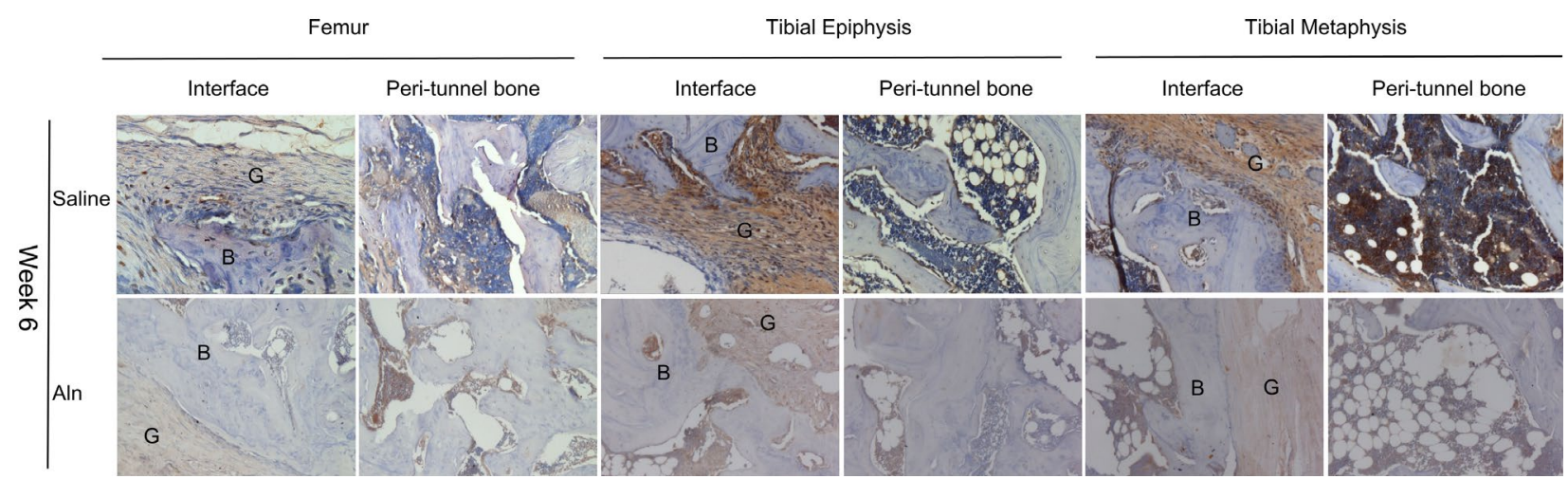

Fig. 14. Representative photomicrographs showing the immunohistochemical staining of MMP1 at different tunnel and peri-tunnel regions in the mid-dose alendronate group and saline group at week 6 after ACL reconstruction. Scale bar $=100 \mu \mathrm{m}$. B: bone; G: graft.

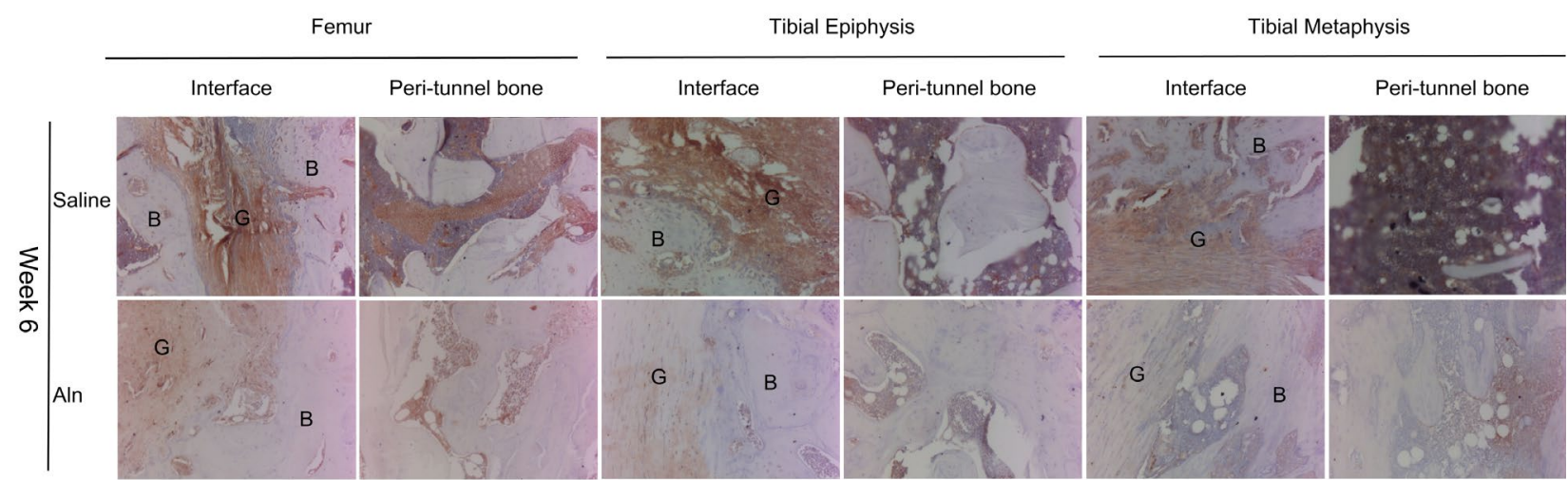

Fig. 15. Representative photomicrographs showing the immunohistochemical staining of MMP13 at different tunnel and peri-tunnel regions in the mid-dose alendronate group and saline group at week 6 after ACL reconstruction. Scale bar $=100 \mu \mathrm{m}$. B: bone; G: graft.

effect of alendronate on tunnel and peri-tunnel bone and week 2-ultimate load and the effect was most marked at the tibial tunnel metaphysis. The effects of alendronate on the improvement of tendon graft to bone tunnel healing were consistent with the results of Thomopoulos et al. (2007) and Hays et al. (2008). Bisphosphonate was reported to suppress bone loss and improved tendonto-bone repair strength in a flexor tendon canine model (Thomopoulos et al., 2007). However, the graft used was not free, as only one end of the tendon was transected. The mechanical environment was thus different from that in
ACL reconstruction. Moreover, the repaired tendon was cut proximally to remove all loads from the distal phalanx repair site during healing and rehabilitation, which reduced the clinical impact. The follow-up time was only 21 days and short. Histological assessment was not performed to explain the mechanisms of changes seen in BMD or biomechanics in their study. Clodronate, a non-nitrogencontaining bisphosphonate was also reported to induce macrophage apoptosis and promoted tendon graft to bone tunnel healing (Hays et al., 2008). The mechanisms of action of nitrogen-containing and non-nitrogen-containing 
bisphosphonates are different. The laxity of medial cruciate ligament (MCL)-complex was also significantly improved after risedronate treatment in an ACL transection-induced osteoarthritis animal model (Doschak et al., 2004; Doschak et al., 2005). Pre-treatment of teeth with alendronate also resulted in better functional restoration of the healing periodontal ligament in replanted teeth (Shibata et al., 2004). The fact that anti-resorptive agents could promote graft-bone healing was also reported in a previous study (Rodeo et al., 2007). They showed that suppression of osteoclastic activity with osteoprotegerin (OPG) increased bone formation while promotion of osteoclastic activity with receptor activator of nuclear factor-kappa B ligand (RANKL) impaired bone ingrowth. The tunnel area was smaller and the stiffness of the bone-graft-bone complex was higher in the OPG group compared to those in the RANKL group (Rodeo et al., 2007). Transforming growth factor (TGF)- $\beta 1$ (Yamazaki et al., 2005), TGF- $\beta 1$ combined with Epidermal Growth Factor (EGF) (Yasuda et al., 2004) and Bone Morphogenetic Protein (BMP)-2 (Rodeo et al., 1999) have been used to promote tendon graft to bone tunnel healing. The effectiveness of alendronate in ACL graft healing could not be compared to these previous studies as different animal models, grafts, tunnel sizes, tunnel orientation, graft fixation methods and follow-up times were used. We reported that alendronate reduced peri-tunnel bone resorption, which has not been reported in other studies using growth factors.

In addition to reducing the number of CD68+ cells and bone resorption at the peri-tunnel, our results showed that alendronate increased mineralised tissue inside bone tunnel and enhanced tunnel graft integrity as well as improved tendon graft to bone tunnel integration. The mechanism of mineralised tissue formation inside the bone tunnel is unclear. We observed a reduction in the expression of MMP1 and MMP13, in addition to CD68-positive cells, at the tendon graft-bone tunnel interface in this study after alendronate treatment. The improvement of intra-tunnel graft integrity and interface healing therefore might be explained by the inhibition of MMP production. Indeed, previous studies showed that bisphosphonates had antiinflammatory effects (Corrado et al., 2007) and could block MMP production (Teronen et al., 1997; Teronen et al., 1999; Nakaya et al., 2000). In another study, inhibition of MMPs improved graft-bone tunnel healing (Demirag et al., 2005).

Our results showed that there was no significant improvement of graft mid-substance remodelling after alendronate treatment and, indeed, the ACL complex failed at graft mid-substances for samples in all groups at week 6 . The graft mid-substance became the weakest point at week 6 and hence the beneficial effect of alendronate at the tunnel interface could not be demonstrated in the biomechanical test at week 6. Despite this, the beneficial effects of alendronate on tunnel bone mass and density, graft-bone tunnel integration and peri-tunnel bone loss were demonstrated by both histology and vivaCT imaging at week 6.

We did not follow up the animals after stopping the application of alendronate. Whether the effect of alendronate could last was not clear. Future long-term studies should also be conducted to monitor the safety and efficacy of long-term dosing of alendronate on tendon graft to bone tunnel healing.

\section{Conclusions}

Alendronate reduced peri-tunnel bone resorption, increased mineralised tissue inside the bone tunnel as well as histologically and biomechanically promoted tendon graft to bone tunnel healing at early stage after ACL reconstruction. Alendronate treatment reduced the expression of MMP1, MMP3 and CD68-positive cells at the peri-tunnel region and graft-bone tunnel interface. Alendronate might be used for reducing peri-tunnel bone loss and promoting graft-bone tunnel healing at early stage post-ACL reconstruction.

\section{Acknowledgements}

The authors would like to thank Mr. Bruma $\mathrm{Fu}$ for establishing the first version of vivaCT image analysis that was later modified for use in this study. This research project was made possible by equipment/resources supported by the GRF (project no. 470808, 08/001/ERG) from University Grant Council and the Hong Kong Jockey Club Charities Trust.

\section{References}

Anderson K, Seneviratne AM, Izawa K, Atkinson BL, Potter HG, Rodeo SA (2001) Augmentation of tendon healing in an intraarticular bone tunnel with use of a bone growth factor. Am J Sports Med 29: 689-698.

Cao YP, Mori S, Mashiba T, Westmore MS, Ma L (2007) Fracture callus under anti-resorptive agent treatment evaluated by pQCT. In Qin L, Genant HK, Griffith J, Leung KS (eds) Advanced bioimaging technologies in assessment of the quality of bone and scaffold materials.- Techniques and applications. Springer, Berlin pp 553-566.

Corrado A, Santoro N, Cantatore FP (2007) Extraskeletal effects of bisphosphonates. Joint Bone Spine 74: 32-38.

Demirag B, Sarisozen B, Ozer O, Kaplan T, Ozturk C (2005) Enhancement of tendon-bone healing of anterior cruciate ligament grafts by blockage of matrix metalloproteinases. J Bone Joint Surg Am 87: 2401-2410.

Ditsios K, Boyer MI, Kusano N, Gelberman RH, Silva MJ (2003) Bone loss following tendon laceration, repair and passive mobilization. J Orthop Res 21: 990-996.

Doschak MR, LaMothe JM, Cooper DM, Hallgrimsson B, Hanley DA, Bray RC, Zernicke RF (2005) Bisphosphonates reduce bone mineral loss at ligament enthuses after joint injury. Osteoarthritis Cartilage 13: 790-797.

Doschak MR, Wohl GR, Hanley DA, Bray RC, Zernicke RF (2004) Antiresoprtive therapy conserves some 
periarticualr bone and ligament mechanical properties after anterior cruciate ligament disruption in the rabbit knee. J Orthop Res 22: 942-948.

Ejerhed L, Kartus J, Nilsen R, Nilsson U, Kullenberg R, Karlsson J (2004) The effect of anterior cruciate ligament surgery on bone mineral in the calcaneus: a prospective study with a 2-year follow-up evaluation. Arthroscopy 20: 352-359.

Fithian DC, Paxton EW, Stone ML, Luetzow WF, csintalan RP, Phelan D, Daniel DM (2005) Prospective trial of a treatment algorithm for the management of the anterior cruciate ligament-injured knee. Am J Sports Med 33: $335-346$.

Fu FH, Bennett CH, Lattermann C, Ma CB (1999) Current trends in anterior cruciate ligament reconstruction. Part 1: Biology and biomechanics of reconstruction. Am J Sports Med 27: 821-830.

Galatz LM, Rothermich SY, Zaegel M, Silva MJ, Havlioglu N, Thomopoulos S (2005) Delayed repair of tendon to bone injuries leads to decreased biomechanical properties and bone loss. J Orthop Res 23: 1441-1447.

George MS, Dunn WR, Spindler KP (2006) Current concepts review: revision anterior cruciate ligament reconstruction. Am J Sports Med 34: 2026-2037.

Giuliani N, Pedrazzoni M, Negri G, Passeri G, Impicciatore M, Girasole G (1998a) Bisphosphonates stimulate formation of osteoblast precursors and mineralized nodules in murine and human bone marrow cultures in vitro and promote early osteoblastogenesis in young and aged mice in vivo. Bone 22: 455-461.

Giuliani N, Pedrazzoni M, Passeri G, Negri G, Impicciatore M, Girasole G (1998b) Bisphosphonates stimulate the production of basic fibroblast growth factor and the formation of bone marrow precursors of osteoblasts. New findings about their mechanism of action. Minerva Med 89: 249-258.

Hays PL, Kawamura S, Deng XH, Dagher E, Mithoefer K, Ying L, Rodeo SA (2008) The role of macrophages in early healing of a tendon graft in a bone tunnel. J Bone Joint Surg Am 90: 565-579.

Kannus P, Sievanen H, Jarvinen M, Heinonen A, Oja P, Vuori I (1992) A cruciate ligament injury produces considerate, permanent osteoporosis in the affected knee. J Bone Miner Res 7: 1429-1434.

Kartus J, Sterner S, Nilsen R, Nilsson U, Eriksson BI, Karisson J (1998) Bone mineral assessments in the calcaneus after anterior cruciate ligament injury. An investigation of 92 male patients before and two years after reconstruction or revision surgery. Scand J Med Sci Sports 8: 449-455.

Leppala J, Kannus P, Natri A, Pasanen M, Sievanen H, Vuori I, Jarvinen M (1999) Effect of anterior cruciate ligament injury of the knee on bone mineral density of the spine and affected lower extremity: a prospective one-year follow-up study. Calcif Tissue Int 64: 357-363.

Lim JK, Hui J, Li L, Thambyah A, Goh J, Lee EH (2004) Enhancement of tendon graft osteointegration using mesenchymal stem cells in a rabbit model for anterior cruciate ligament reconstruction. Arthroscopy 20: 899-910.

Lui PPY, Chan LS, Fu SC, Chan KM(2010a) Expression of sensory neuropeptides in tendon is associated with failed healing and activity-related tendon pain in collagenaseinduced tendon injury. Am J Sports Med 38: 757-764.

Lui PP, Ho G, Shum WT, Lee YW, Ho PY, Lo WN, Lo $\mathrm{CK}$ (2010b) Inferior tendon graft to bone tunnel healing at the tibia compared to that at the femur after anterior cruciate ligament reconstruction. J Orthop Sci 15: 389-401.

Lui PPY, Ho G, Lee YW, Ho PY, Lo WN, Lo CK (2011) Validation of a histologic scoring system for the examination of quality of tendon graft to bone tunnel healing in ACL reconstruction: TBTH Score (Tendon-Bone Tunnel Healing) Score. Anal Quant Cytol Histol 33: 36-49.

Lui PPY, Cheng YY, Yung SH, Hung ASL, Chan KM (2012) A randomized controlled trial comparing bone mineral density changes of three different ACL reconstruction techniques. Knee 19: 779-785.

Ma CB, Kawamura S, Deng XH, Ying L, Schneidkraut J, Hays P, Rodeo SA (2007) Bone morphogenetic proteinssignaling plays a role in tendon-to-bone healing: a study of rhBMP-2 and noggin. Am J Sports Med 3: 597-604.

Ma YL, Bryant HU, Zeng QQ, Schmidt A, Hoover J, Cole HW, Yao W, Jee WSS, Sato M (2003) New bone formation with teriparatide [human parathyroid hormone-91-340] is not retarded by long-term pretreatment with alendronate, estrogen, or raloxifene in ovariectomized rats. Endocrinology 144: 2008-2015.

Nakaya H, Osawa G, Iwasaki N, Cochran DL, Kamoi K, Oates TW (2000) Effects of bisphosphonate on matrix metalloproteinase enzymes in human periodontal ligament cells. J Periodontol 71: 1158-1166.

Ohtera K, Yamada Y, Aoki M, Sasaki T, Yamakoshi K (2000) Effects of periosteal wrapped around tendon in a bone tunnel: a biomechanical and histological study in rabbits. Crit Rev Biomed Eng 28: 115-118.

Rittweger J, Maffulli N, Maganaris CN, Narici MV (2005) Reconstruction of the anterior cruciate ligament with a patella-tendon-bone graft may lead to a permanent loss of bone mineral content due to decreased patellar tendon stiffness. Med Hypotheses 64: 1166-1169.

Rodeo SA, Kawamura S, Ma BC, Deng XH, Sussman PS, Hays P, Ying L (2007) The effect of osteoclastic activity on tendon-to-bone healing: An experimental study in rabbits. Am J Bone Joint Surg Am 89: 2250-2259.

Rodeo SA, Suzuki K, Deng XH, Wozney J, Warren RF (1999) Use of recombinant human bone morphogenetic protein-2 to enhance tendon healing in a bone tunnel. Am J Sports Med 27: 476-488.

Shibata T, Komatsu K, Shimada A, Shimoda S, Oida S, Kawasaki K, Chiba M (2004) Effects of alendronate on restoration of biomechanical properties of periodontium in replanted rat molars. J Periodontal Res 39: 405-414.

Silva MJ, Boyer MI, Ditsios K, Burns ME, Harwood FL, Amiel D, Gelberman RH (2002) The insertion site of the canine flexor digitorum profundus tendon heals slowly following injury and suture repair. J Orthop Res 20: 447453.

Teronen O, Heikkila P, KonttinenYT, Laitinen M, Salo T, Hanemaaijer R, Teronen A, Maisi P, Sorsa T (1999) MMP inhibition and downregulation by bisphosphonates. Ann N Y Acad Sci 878: 453-465.

Teronen O, Konttinen YT, Lindqvist C, Salo T, Ingman T, Lauhio A, Ding Y, Santavirta S, Sorsa T (1997) Human 
neutrophil collagenase MMP-8 in peri-implant sulcus fluid and its inhibition by chodronate. J Dent Res 76: 1529-1537.

Thomopoulos S, Matsuzaki H, Zaegel M, Gelberman RH, Silva MJ (2007) Alendronate prevents bone loss and improves tendon-to-bone repair strength in a canine model. J Orthop Res 25: 473-479.

von Knoch F, Jaquiery C, Kowalsky M, Schaeren S, Alabre C, Martin I, Rubash HE, Shanbhag AS (2005) Effects of bisphosphonates on proliferation and osteoblast differentiation of human bone marrow stromal cells. Biomaterials 26:6941-6949.

Wang CJ, Wang FS, Yang KD, Weng CH, Sun YC, Yang YJ (2005) The effect of shock wave treatment at the tendon-bone interface-an histomorphological and biochemical study in rabbits. J Orthop Res 23: 274-280.

Weiler A, Peine R, Pashmineh-Azar A, Abel C, Sudkamp NP, Hoffmann RF (2002) Tendon healing in a bone tunnel. Part I: Biomechanical results after biodegradable interference fit fixation in a model of anterior cruciate ligament reconstruction in sheep. Arthroscopy 18: 113-123.

Wen CY, Qin L, Lee KM, Wong MW, Chan KM (2009) Influence of bone adaption on tendon-to-bone healing in bone tunnel after anterior cruciate ligament reconstruction in a rabbit model. J Orthop Res 27: 1447-1456.

Yamazaki S, Yasuda K, Tomita F, Tohyama H, Minami A (2005) The effect of transforming growth factor-beta 1 on intraosseous healing of flexor tendon allograft replacement of anterior cruciate ligament in dogs. Arthroscopy 21: 1034-1041.

Yasuda K, Tomita F, Yamazaki S, Minami A, Tohyama $H$ (2004) The effect of growth factors on biomechanical properties of the bone-patellar tendon-bone graft after anterior cruciate ligament reconstruction: a canine model study. Am J Sports Med 32: 870-880.

Yunes M, Richmond JC, Engles EA, Pinczewski LA (2001) Patellar versus hamstring tendons in anterior cruciate ligament reconstruction: a meta-analysis. Arthroscopy 17: 248-257.

Zerahn B, Munk AO, Helweg J, Hovgaard C (2006) Bone mineral density in the proximal tibia and calcaneus before and after arthroscopic reconstruction of the anterior cruciate ligament. Arthroscopy 22: 265-269.

\section{Discussion with Reviewers}

Reviewer I: Can the authors give a comment on the clinical relevance of their study? Is the observed effect sufficient with respect to ACL reconstruction?

Authors: Our results showed that alendronate reduced peri-tunnel bone resorption, increased mineralised tissue inside the bone tunnel as well as histologically improved tendon graft to bone tunnel healing at both week 2 and week 6 after ACL reconstruction. This resulted in the improvement of ultimate load of the healing complex at week 2 but not at week 6 after ACL reconstruction when the weak link shifted to graft mid-substance at week 6 . Early stability of knee joint due to improved tendon graft to bone tunnel healing might allow the patients to adopt a more aggressive rehabilitation therapy and hence may improve the long-term outcome after surgery. Peri-tunnel bone loss may increase the risk of post-operative fractures. Indeed, post-operative fracture due to low-energy trauma is a severe, though rare, complication of ACL reconstruction. Peri-tunnel bone loss may also complicate ACL revision surgery (please see our reply to another reviewer). Administration of alendronate at the initial stage hence may benefit patients undergoing ACL reconstruction.

Reviewer I: What is the advantage as compared to other treatments, like augmentation with growth factors.

Authors: This is difficult to compare the findings from different laboratories as different animal models, grafts, tunnel size, tunnel orientation, graft fixation methods, and follow-up times were used. We found that alendronate could reduce peri-tunnel bone resorption which has not been reported in other studies using growth factors. Alendronate is an approved drug for the treatment of osteoporosis. The efficacy of alendronate in the promotion of ACL graft healing might facilitate its clinical use compared to the growth factors reported in the previous studies.

Reviewer II: Recently, a number of negative outcomes associated with bisphosphonates have been reported, including brittle bones and osteonecrosis. Are these risks appropriate for improving ACL reconstruction outcomes? ACL injuries are typically sports-related and in a young population, so care must be taken with potential long-term side effects.

Authors: Osteonecrosis of the jaw (ONJ) has been reported after long-term use of bisphosphonates. However, it is uncommon and occurs primarily in patients being administered high-dose intravenous bisphosphonate, with most cases being reported in cancer patients (Kuhl et al., 2012, additional reference). According to a recently published systematic review (Kuhl et al., 2012), the mean incidence of bisphosphonate-related ONJ on oral bisphosphonate of 9 studies was $0.12 \%(0-4.3 \%)$. When bisphosphonate was intravenously administered in oncologic dosage, the incidence of ONJ increased. The mean incidence over a total of 47 studies on intravenous application was $7 \%(0-27.5 \%)$. Previous studies also showed that people on long-term use of alendronate for more than 5 years are at risk of developing low-impact femoral fractures (Kwek et al., 2008, additional reference). Most of the patients having ACL reconstructions are young and they are expected to be on alendronate treatment for a limited time post-reconstruction when peri-tunnel bone resorption occurs and tendon graft to bone tunnel healing is critical to the clinical outcome. Peri-tunnel bone loss has several implications for tendon graft to bone-tunnel healing. First, the tunnel surface might become less stable for tendon-bone integration due to increased bone resorption. BMD decrease might also complicate revision surgery and may be even fracture due to the reduction in bone strength. Indeed, postoperative fracture is a serious, albeit rare, complication after ACL reconstruction. There were case reports of femoral condyle fractures (Wiener and Siliski, 1996; Wilson et al., 2004; Montgomery and Evans, 2008, additional references) and proximal tibial 
fractures (Morgan and Steensen, 1998; Mithofer et al., 2004; Salem et al., 2007, additional references) after ACL reconstruction. All these injuries occurred through low energy trauma and most were sustained within the first few months after reconstruction. Our results showed that alendronate reduced peri-tunnel bone resorption and promoted tendon graft to bone tunnel healing. Although alendronate was shown to significantly improve ultimate load of the healing complex at week 2 , but not at week 6 when the weak link shifted to the graft mid-substance, early stability of knee joint due to improved tendon graft to bone tunnel healing might allow the patient to adopt more aggressive rehabilitation therapy. Therefore, we believe that the risks are appropriate for improving ACL reconstruction outcomes.

Reviewer II: What dosage schedule would this study imply for patients? Should alendronate be given immediately after surgery or immediately after injury? Is a short time course appropriate to reduce the risk of negative side effects?

Authors: Based on the result of this study, we would recommend taking the middle-dose of subcutaneous alendronate injection $(60 \mu \mathrm{g} / \mathrm{kg} / \mathrm{week})$ for a limited period at the early stage post-ACL reconstruction when peritunnel bone resorption occurs and when tendon graft to bone tunnel healing is critical in determining the clinical outcome. We have not tested the effect of alendronate on the prevention of bone resorption after injury, though a small but statistically significant bone loss at the patellar and proximal tibia of the ACL injured knee of conservatively treated patients has been reported (Leppala et al., 1999, text reference). This requires another study. As considerable and statistically significant bone loss occurred after ACL reconstruction (Leppala et al., 1999; Lui et al., 2012, text references), alendronate treatment should be administered after ACL reconstruction. As the duration of alendronate treatment is expected to be limited, we believe that a short time course is appropriate to reduce the risk of negative side effects.

Reviewer II: The mechanical improvements were not very dramatic. Are small increases in failure load (only at an early time point) clinically meaningful?

Authors: The observed improvement in peri-tunnel bone and increase in tendon graft to bone tunnel healing is seen up to week 6 after alendronate treatment. However, since alendronate has no effect on graft mid-substance remodelling, significant improvement in ultimate load was only observed at week 2 but not at week 6 , when the weak-link shifted to the graft mid-substance. As mentioned above, peri-tunnel bone loss has several implications on the outcome of ACL reconstruction and early stability of knee joint due to improved tendon graft to bone tunnel healing might allow the patients to adopt more aggressive rehabilitation therapy. Therefore, we believe that the advantage of administering alendronate at the early stage post-ACL reconstruction is clinically meaningful.

Reviewer II: Am I wrong to suggest that if all ACLs had failed at 6 weeks the measurements provided actually study more the level of initial bone absorption, not bony healing? Is the degree of bone absorption directly proportional to the end stage of bony healing?

Authors: We measured the mass and density of (1) newly-formed mineralised tissue inside bone tunnel and (2) peri-tunnel bone. The ROI for measurement is shown in Fig. 1.We measured bone formation inside bone tunnel and bone resorption at the peri-tunnel region at baseline and at different times after ACL reconstruction. Hence, we confirmed that bone resorption occurred after ACL reconstruction. Regarding the effect of peri-tunnel bone resorption on bone formation inside bone tunnel and the mechanical strength of the bone-tendon-bone complex, our unpublished results showed that there was significant negative correlation between peri-tunnel BMD and tunnel BMD (rho $=-0.204 ; p=0.034)$, but not between peri-tunnel $\mathrm{BV} / \mathrm{TV}$ and tunnel BV/TV (rho $=0.121 ; p=0.214$ ). The ultimate load and stiffness of the healing complex positively correlated with tibial tunnel bone formation and negatively correlated with tibial peri-tunnel bone, especially at the metaphyseal segment. Therefore, it seemed that the loss of peri-tunnel bone did not deterministically influence the early stage of tunnel healing. While peri-tunnel bone loss might not directly contribute to the healing quality of the complex at the early stage, it might undermine the support of graft-bone tunnel complex, complicates revision surgery or causes fracture due to the reduction in bone strength. Indeed, postoperative fracture is a serious, albeit, rare complication after ACL reconstruction. There were case reports of femoral condyle fractures (Wilson et al., 2004; Thangamani et al., 2009, additional references) and proximal tibial fractures (Mithofer et al., 2004; Salem et al., 2007, additional references) after ACL reconstruction. All these injuries occurred through low energy trauma and most were sustained within the first several months after reconstruction. We recommend protection of the knee joint at the early stage after ACL reconstruction due to the inherent weakness of the bone around the knee and prevention of peri-tunnel bone loss by alendronate as shown in this study. Besides reducing peri-tunnel bone loss, our results further showed that alendronate increased the mass and density of mineralised tissue inside the bone tunnel and increased the strength the bone-tendon-bone construct at the early stage after ACL reconstruction. Therefore, alendronate also has positive effect on tendon graft to bone tunnel healing after ACL reconstruction.

\section{Additional References}

Kuhl S. Walter C, Acham S, Pfeffere R, Lambrecht JT (2012) Bisphosphonate-related osteonecrosis of the jaws - a review. Oral Oncol 48: 938-947.

Kwek E, Goh S, Koh J, Png M, Howe T (2008) An emerging pattern of subtrochanteric stress fractures: A long-term complication of alendronate therapy. Injury 39: 224-231.

Mithofer K, Gill TJ, Vrahas MS (2004) Tibial plateau fracture following anterior cruciate ligament reconstruction. Knee Surg Sports Traumatol Arthrosc 12: 325-328. 
Montgomery CO, Evans RP (2008) Arthroscopic reduction and internal fixation of a medial femoral condylar fracture after anterior cruciate ligament reconstruction. A case report. J Bone Joint Surg Am 90: 863-868.

Morgan E, Steensen RN (1998) Traumatic proximal tibial fracture following anterior cruciate ligament reconstruction. Am J Knee Sports Traumatol Arthrosc 11: 193-194.

Salem K, Rees DC, Geutjens G (2007) Low velocity bicondylar tibial fracture following ACL reconstruction. Injury Extra 38:179-181.
Thangamani VB, Flanigan DC, Merk BR (2009) Intraarticular distal femur fracture extending from an expanded femoral tunnel in an anterior cruciate ligament (ACL) reconstructed knee: a case report. J Trauma 67: E209-E212.

Wiener DF, Siliski JM (1996) Distal femoral shaft fracture: a complication of endoscopic anterior cruciate ligament reconstruction. A case report. Am J Sports Med 24: 244-247.

Wilson TC, Rosenblum WJ, Johnson DL (2004) Fractrue of the femoral tunnel after an anterior cruciate ligament reconstruction. Arthroscopy 20: e45-e47. 